ITP-SB-93-84

BONN-HE-93-49

December 1993

hep-th/9312049

\title{
A class of $\mathcal{W}$-algebras with infinitely generated classical limit
}

\author{
J. de Boer ${ }^{1}$, L. Fehér ${ }^{2, *}$ and A. Honecker ${ }^{2}$ \\ ${ }^{1}$ Institute for Theoretical Physics \\ State University of New York at Stony Brook \\ Stony Brook, NY 11794-3840, USA \\ ${ }^{2}$ Physikalisches Institut der Universität Bonn \\ Nussallee 12, 53115 Bonn, Germany
}

\begin{abstract}
There is a relatively well understood class of deformable $\mathcal{W}$-algebras, resulting from Drinfeld-Sokolov (DS) type reductions of Kac-Moody algebras, which are Poisson bracket algebras based on finitely, freely generated rings of differential polynomials in the classical limit. The purpose of this paper is to point out the existence of a second class of deformable $\mathcal{W}$-algebras, which in the classical limit are Poisson bracket algebras carried by infinitely, nonfreely generated rings of differential polynomials. We present illustrative examples of coset constructions, orbifold projections, as well as first class Hamiltonian reductions of DS type $\mathcal{W}$-algebras leading to reduced algebras with such infinitely generated classical limit. We also show in examples that the reduced quantum algebras are finitely generated due to quantum corrections arising upon normal ordering the relations obeyed by the classical generators. We apply invariant theory to describe the relations and to argue that classical cosets are infinitely, nonfreely generated in general. As a by-product, we also explain the origin of the previously constructed and so far unexplained deformable quantum $\mathcal{W}(2,4,6)$ and $\mathcal{W}(2,3,4,5)$ algebras.
\end{abstract}

\footnotetext{
* An Alexander von Humboldt Fellow. On leave from Bolyai Institute of Szeged University, H-6720 Szeged, Hungary.
} 


\section{Introduction}

The classification of extended conformal algebras (also called $\mathcal{W}$-algebras, or local chiral algebras) is a key ingredient to the classification of two-dimensional rational conformal field theories, which apart from being interesting in its own right is also interesting since it is related to problems in statistical physics, string theory and integrable systems. The experience accumulated so far (see [1] for a review) shows that $\mathcal{W}$-algebras come in two varieties. Firstly, there exist 'deformable' (or 'generic') $\mathcal{W}$-algebras for which the structure constants are continuous functions — with isolated singularities — of the Virasoro centre $c$ for a fixed field content, i.e., for a fixed conformal weight spectrum of generating fields. Secondly, there exist also 'nondeformable' (or 'exceptional') $\mathcal{W}$-algebras that appear only at particular, isolated $c$ values. It is generally believed that, with notable exceptions, the nondeformable $\mathcal{W}$-algebras can be understood in terms of the deformable ones, for instance they could occur in particular minimal models of deformable $\mathcal{W}$-algebras. Most deformable $\mathcal{W}$-algebras considered so far result from Drinfeld-Sokolov (DS) type Hamiltonian reductions of affine Kac-Moody algebras, and thus have a classical limit which is a Poisson bracket algebra carried by a differential polynomial ring generated by a finite number of independent generating fields. To put it differently, a class of deformable quantum $\mathcal{W}$-algebras exists to which those algebras belong which admit a finitely, freely generated classical limit. It appears that this class of $\mathcal{W}$-algebras is by now reasonably well understood (see, e.g., [2-5] and references therein), though a lot of work remains to be done before we will have it completely catalogued.

However, there are a number of reasons for believing that the above mentioned class does not exhaust the deformable quantum $\mathcal{W}$-algebras. We have such indications in the context of each three methods usually used for obtaining $\mathcal{W}$-algebras; the direct constructions, coset constructions and the first class Hamiltonian reduction method. Indeed, the direct constructions provided two so far unexplained deformable $\mathcal{W}$-algebras, with conformal weights $2,4,6[6,7]$ and $2,3,4,5$ [8], respectively, for which the procedure used in [2] for extracting the classical limit fails. In this paper we shall explain these algebras in terms of certain coset constructions, and shall see that their classical analogues are in fact infinitely, nonfreely generated (and therefore these algebras would appear to have no classical limit if one tries to force the procedure of [2] which is geared towards a finitely generated classical limit). Examples of classically infinitely generated coset algebras have recently been discussed in [9]. More generally, there is a large class of coset algebras, including for instance

the diagonal cosets $\left(\widehat{\mathcal{G}}_{k} \oplus \widehat{\mathcal{G}}_{m}\right) / \widehat{\mathcal{G}}_{k+m}$ at generic levels $k$ and $m$, for which a simple group theoretic argument given in this paper shows that generically they have infinitely generated classical limits. Finally, it is clear that the DS reductions, which underlie all known deformable $\mathcal{W}$-algebras with a finitely, freely generated classical limit, are a very special 
subclass of the reductions of Kac-Moody algebras defined by conformally invariant first class constraints. In some examples of first class reductions to which the DS mechanism does not apply it has already been shown in [4] that the ring of gauge invariant differential polynomials is not freely generated. In this paper we further develop an illustrative example of this sort and demonstrate that the invariant ring carrying the reduced Poisson bracket algebra is infinitely, nonfreely generated.

We wish to emphasize that although reductions of finitely generated DS type $\mathcal{W}$ algebras and Kac-Moody algebras seem to lead generically to infinitely, nonfreely generated algebras at the classical level, the corresponding reduced quantum algebras are finitely generated in all cases studied so far ${ }^{1}$. We shall see that the underlying mechanism responsible for this is that the infinitely many classical generators are not independent but obey infinitely many relations and upon normal ordering (a subset of) the relations quantum corrections arise which allow for eliminating the infinitely many 'would-be-generators' in favour of a finite subset.

The paper is organized as follows. We shall first analyze a very simple example, a reduction of a $\beta-\gamma$ system, in detail to illustrate the ideas. In particular, we shall see why the quantum version of the classically infinitely generated reduced algebra is finitely generated. Then we shall explain how this construction is related to a coset construction underlying the so far unexplained $\mathcal{W}(2,4,6)$-algebra. This will lead us to discussing general coset constructions as well as bosonic projections of fermionic $\mathcal{W}$-algebras and orbifolds of $\mathcal{W}$-algebras that also possess infinitely, nonfreely generated classical analogues in general. Finally, to show that infinitely, nonfreely generated classical algebras arise in all reduction procedures, we treat an example of Hamiltonian reduction by first class constraints that leads to such a reduced classical system. We give our conclusions and comment on open problems at the end of the main text, and there are also two appendices containing technical material.

\section{A reduction of a $\beta-\gamma$ system}

Below, we work out our simplest example, first at the classical then at the quantum level. We start by considering two independent generating fields $\xi_{ \pm}(z)$ defined on the circle, subject to the Poisson brackets (PBs):

$$
\left\{\xi_{-}(x), \xi_{+}(y)\right\}=\delta(x-y), \quad\left\{\xi_{-}(x), \xi_{-}(y)\right\}=\left\{\xi_{+}(x), \xi_{+}(y)\right\}=0
$$

Then we have the chiral algebra carried by the differential ring $\mathcal{P}$ consisting of the polynomials in $\xi_{ \pm}^{(i)}:=\partial^{i} \xi_{ \pm}$. The fields $\xi_{ \pm}$are primary fields of weight $\frac{1}{2}$ with respect to the

\footnotetext{
1 A claim to the contrary made in ref. [10] is not correct, see also section 3.
} 
conformal structure defined by

$$
T:=-\frac{1}{2}\left(\xi_{+} \partial \xi_{-}-\xi_{-} \partial \xi_{+}\right) .
$$

This system is the classical version of a linear ' $\beta-\gamma$ system' $\left(\beta:=\xi_{-}, \gamma:=\xi_{+}\right)$often used in conformal field theory. We introduce the $\widehat{s l(2)}$ subalgebra of $\mathcal{P}$ generated by the currents

$$
J_{H}:=\xi_{-} \xi_{+}, \quad J_{E}:=-\frac{1}{2} \xi_{+}^{2}, \quad J_{F}:=\frac{1}{2} \xi_{-}^{2},
$$

satisfying the PB relations

$$
\begin{aligned}
& \left\{J_{H}(x), J_{E}(y)\right\}=2 J_{E}(y) \delta, \quad\left\{J_{H}(x), J_{F}(y)\right\}=-2 J_{F}(y) \delta, \quad\left\{J_{E}(x), J_{F}(y)\right\}=J_{H}(y) \delta \\
& \left\{J_{H}(x), \xi_{ \pm}(y)\right\}= \pm \xi_{ \pm}(y) \delta, \quad\left\{J_{E}(x), \xi_{-}(y)\right\}=\xi_{+}(y) \delta, \quad\left\{J_{F}(x), \xi_{+}(y)\right\}=\xi_{-}(y) \delta
\end{aligned}
$$

where $\delta=\delta(x-y)$. We wish to describe the reduced chiral algebra carried by the commutant (centralizer) $\mathcal{P}_{s l(2)}$ of the $s l(2)$ defined by the zero modes of the currents (1.3) in $\mathcal{P}$. In other words, we are interested in the 'classical coset' of the $\beta$ - $\gamma$ algebra with respect to the 'horizontal' subalgebra $s l(2) \subset \widehat{s l(2)} \subset \mathcal{P}$, i.e., $\mathcal{P}_{s l(2)}=\mathcal{P} / \operatorname{sl}(2)$.

To find the commutant notice from (1.4) that $\mathcal{P}$ is a ring of polynomials in infinitely many variables that form doublets, $\left(\xi_{+}^{(i)}, \xi_{-}^{(i)}\right)$ for any $i=0,1, \ldots$, under the global $s l(2)$ transformations generated by the zero modes of the currents. Therefore the pairwise symplectic scalar products of the doublets, given by

$$
W_{i, j}:=\xi_{+}^{(i)} \xi_{-}^{(j)}-\xi_{-}^{(i)} \xi_{+}^{(j)},
$$

are obviously $\operatorname{sl}(2)$ invariants, i.e., belong to the invariant subring $\mathcal{P}_{s l(2)} \subset \mathcal{P}$. The fact that the $W_{i, j}$ are actually a generating set of $\mathcal{P}_{s l(2)}$ is much less obvious, but it follows from invariant theory. In the terminology of Weyl [11], this is just the 'first main theorem' of invariant theory for the (defining representation of the) classical group $S L(2)$.

It is perhaps worth recalling here that invariant theory (see, e.g., $[11,12]$ ) deals with the following class of problems (among others). Take a group $G$ and a set $V_{\alpha}, \ldots, V_{\omega}$ of finite dimensional representations of $G$. Consider the space $\mathcal{M}$ of polynomials $p$ depending on (a fixed or arbitrary number of) variables that belong to these representations, $p=$ $p\left(v_{\alpha}^{0}, \ldots, v_{\alpha}^{m_{\alpha}}, \ldots, v_{\omega}^{0}, \ldots, v_{\omega}^{m_{\omega}}\right)$, where $v_{\alpha}^{i_{\alpha}} \in V_{\alpha}$ for $i_{\alpha}=0, \ldots, m_{\alpha}$ etc. The linear space $\mathcal{M}$ carries a natural representation of $G$ induced by transforming the arguments of $p$. The problem then is to describe the $G$-singlets, i.e., the invariant polynomials. An important point is that since $\mathcal{M}$ is a polynomial ring so is the subring of invariants. The two major questions are: i) Describe a generating set of the invariant ring. ii) Find the relations obeyed by the (in general not algebraically independent) generators. The answer to i) is called the 
'first main theorem' and the answer to ii) the 'second main theorem' of invariant theory for a given problem. In particular, in [11] these problems are solved for $G$ a classical group and $\mathcal{M}$ the space of polynomials depending on an arbitrary number of variables in the vector (defining) representation of $G$.

Returning to our problem, it is easy to check that in addition to antisymmetry,

$$
W_{i, j}+W_{j, i}=0,
$$

the generators $W_{i, j}$ satisfy the following relation:

$$
W_{i, j} W_{k, l}-W_{i, k} W_{j, l}+W_{i, l} W_{j, k}=0,
$$

for any $i, j, k, l$. The relation (1.7) is known as the 'syzygy' in invariant theory, and the 'second main theorem' of invariant theory states that all polynomial relations between the $s l(2)$ invariant polynomials are consequences of (1.6) and (1.7) (see Chapter VI.1 in [11] and also Corollary 3.2 in [13]). Of course, this statement holds if we consider the variables $\xi_{ \pm}^{(i)}$ independent, i.e., if we forget about the action of the derivation $\partial$ of the differential ring $\mathcal{P}$, given by $\partial \xi_{ \pm}^{(i)}=\xi_{ \pm}^{(i+1)}$. Taking the derivation into account we also have the relation

$$
\partial W_{i, j}=W_{i+1, j}+W_{i, j+1} .
$$

The generating set $\left\{W_{i, j}\right\}$ is overcomplete (redundant) on account of (1.8) and to describe the reduced PB algebra in the most economical way we should select a subset of the generators forming a minimal generating set of $\mathcal{P}_{s l(2)}$. For this consider the linear span of the redundant set of generators at scale dimension $d$ :

$$
\mathcal{V}_{d}:=\text { linear } \operatorname{span}\left\{W_{i, j} \mid i+j+1=d\right\} \text {. }
$$

The derivation $\partial$ maps $\mathcal{V}_{d}$ into $\mathcal{V}_{d+1}$ according to (1.8) and it is easy to see that by using this we can express the generators $\left\{W_{i, j} \mid \forall i, j\right\}$ in terms of a subset of the generators spanning one dimensional subspaces of $\mathcal{V}_{2 r}$ for $r=1,2, \ldots$ any natural number. In this way we obtain a nonredundant (minimal) generating set of $\mathcal{P}_{s l(2)}$, for instance $\left\{W_{1,2 s}\right\}$ with $s$ running over the nonnegative integers. In terms of the redundant generating set the reduced chiral algebra is given by

$$
\begin{aligned}
\left\{W_{i, j}(x), W_{k, l}(y)\right\} & =(-1)^{i+1} \sum_{a=0}^{i+l}(-1)^{a}\left(\begin{array}{c}
i+l \\
a
\end{array}\right) W_{k, i+j+l-a}(y) \delta^{(a)}(x-y) \\
& +(-1)^{j} \sum_{b=0}^{j+l}(-1)^{b}\left(\begin{array}{c}
j+l \\
b
\end{array}\right) W_{k, i+j+l-b}(y) \delta^{(b)}(x-y) \\
& +(-1)^{j+1} \sum_{c=0}^{j+k}(-1)^{c}\left(\begin{array}{c}
j+k \\
c
\end{array}\right) W_{l, i+j+k-c}(y) \delta^{(c)}(x-y) \\
& +(-1)^{i} \sum_{d=0}^{i+k}(-1)^{d}\left(\begin{array}{c}
i+k \\
d
\end{array}\right) W_{l, i+j+k-d}(y) \delta^{(d)}(x-y) .
\end{aligned}
$$


In the present example the r.h.s. is linear in the generators and we observe that this feature is also valid for the PB's of the generators $\left\{W_{1,2 s}\right\}$, as the elements of the redundant generating set are given by linear expressions in terms of the nonredundant generating set $\left\{W_{1,2 s}\right\}$. The elements in the nonredundant generating set $\left\{W_{1,2 s}\right\}$ are of course not independent, since the number of degrees of freedom should not increase in a reduction and we started with just two generating fields $\xi_{ \pm}$. Indeed, they satisfy the infinitely many differential-algebraic relations that can be obtained from $(1.6),(1.7)$ and (1.8) by expressing all $W_{i, j}$ in terms of $\left\{W_{1,2 s}\right\}$.

The main result of the above analysis is that infinitely many generators obeying infinitely many differential-algebraic relations are needed to describe the reduced classical chiral algebra carried by $\mathcal{P}_{s l(2)}$. We next analyze this reduction at the quantum level. For this we take two bosonic chiral quantum fields $\hat{\xi}_{+}(z), \hat{\xi}_{-}(z)$, where the argument now varies on the punctured complex plane, for which the only nonregular OPE is

$$
\hat{\xi}_{-}(x) \hat{\xi}_{+}(y):=\frac{\hbar}{x-y}+\operatorname{reg}
$$

Here we introduced the Planck constant $\hbar$ explicitly in order to make clear the correspondence to the PB (1.1), but one could of course set $\hbar$ to unity by a rescaling of the fields. The fields $\hat{\xi}_{ \pm}$are primary fields of weight $\frac{1}{2}$ with respect to the conformal structure defined by

$$
\hat{T}:=-\frac{1}{2}\left(\hat{\xi}_{+} \partial \hat{\xi}_{-}-\hat{\xi}_{-} \partial \hat{\xi}_{+}\right)
$$

We adopted the notation of Bais et al [14], thus $(A B)$ denotes the usual normal ordered product of the fields $A$ and $B$. We also define the quantum $\widehat{s l(2)}$ currents by normal ordering the expressions in (1.3),

$$
\hat{J}_{H}:=\left(\hat{\xi}_{-} \hat{\xi}_{+}\right), \quad \hat{J}_{E}:=-\frac{1}{2}\left(\hat{\xi}_{+} \hat{\xi}_{+}\right), \quad \hat{J}_{F}:=\frac{1}{2}\left(\hat{\xi}_{-} \hat{\xi}_{-}\right)
$$

and find the (only nonregular) OPE's of the quantum currents to be

$$
\begin{aligned}
& \hat{J}_{H}(x) \hat{J}_{E}(y)=\frac{2 \hbar \hat{J}_{E}(y)}{x-y}+\text { reg. } \\
& \hat{J}_{H}(x) \hat{J}_{F}(y)=\frac{-2 \hbar \hat{J}_{F}(y)}{x-y}+\text { reg. } \\
& \hat{J}_{H}(x) \hat{J}_{H}(y)=\frac{-\hbar^{2}}{(x-y)^{2}}+\text { reg. } \\
& \hat{J}_{E}(x) \hat{J}_{F}(y)=-\frac{1}{2} \frac{\hbar^{2}}{(x-y)^{2}}+\frac{\hbar \hat{J}_{H}(y)}{x-y}+\text { reg. }
\end{aligned}
$$


which (by putting $\hbar=1$ ) is $\widehat{s l(2)}$ at level $-\frac{1}{2}$. The fields $\hat{\xi}_{ \pm}$generate the chiral algebra $\hat{\mathcal{P}}$, i.e., the linear space $\hat{\mathcal{P}}$ is spanned by the (repeated) derivatives and normal ordered products of $\hat{\xi}_{ \pm}$. The object of our interest is the coset

$$
\hat{\mathcal{P}}_{s l(2)}:=\frac{\hat{\mathcal{P}}}{s l(2)},
$$

i.e., the set of fields in $\hat{\mathcal{P}}$ that commute with the horizontal subalgebra $\operatorname{sl}(2) \subset \widehat{s l(2)} \subset \hat{\mathcal{P}}$ spanned by the charges

$$
Q_{A}=\frac{1}{2 \pi i} \oint d x \hat{J}_{A}(x), \quad A=E, F, H
$$

It is straightforward to verify that the quantum versions of the invariants in (1.5), given by

$$
\hat{W}_{i, j}:=\left(\hat{\xi}_{+}^{(i)} \hat{\xi}_{-}^{(j)}\right)-\left(\hat{\xi}_{-}^{(i)} \hat{\xi}_{+}^{(j)}\right), \quad \hat{\xi}_{ \pm}^{(i)}:=\partial^{i} \hat{\xi}_{ \pm},
$$

belong to $\hat{\mathcal{P}}_{s l(2)}$. In the classical limit $\hat{\mathcal{P}}_{s l(2)}$ becomes the differential ring $\mathcal{P}_{s l(2)}$ and we know that the vector space $\mathcal{P}_{s l(2)}$ is spanned by the (repeated) derivatives and products of the $W_{i, j}$. From this we can conclude that $\hat{\mathcal{P}}_{s l(2)}$ is spanned by the derivatives and normal ordered products of the $\hat{W}_{i, j}$. The OPE's of the generating set $\left\{\hat{W}_{i, j}\right\} \subset \hat{\mathcal{P}}_{s l(2)}$ read

$$
\begin{aligned}
\hat{W}_{i, j}(x) \hat{W}_{k, l}(y) & =(-1)^{i+1} \hbar \sum_{a=0}^{i+l}\left(\begin{array}{c}
i+l \\
a
\end{array}\right) \hat{W}_{k, i+j+l-a}(y) \partial_{y}^{a} \frac{1}{x-y} \\
& +(-1)^{j} \hbar \sum_{b=0}^{j+l}\left(\begin{array}{c}
j+l \\
b
\end{array}\right) \hat{W}_{k, i+j+l-b}(y) \partial_{y}^{b} \frac{1}{x-y} \\
& +(-1)^{j+1} \hbar \sum_{c=0}^{j+k}\left(\begin{array}{c}
j+k \\
c
\end{array}\right) \hat{W}_{l, i+j+k-c}(y) \partial_{y}^{c} \frac{1}{x-y} \\
& +(-1)^{i} \hbar \sum_{d=0}^{i+k}\left(\begin{array}{c}
i+k \\
d
\end{array}\right) \hat{W}_{l, i+j+k-d}(y) \partial_{y}^{d} \frac{1}{x-y} \\
& +2 \hbar^{2} \frac{(-1)^{i+j+1}[(i+l) !(j+k) !-(i+k) !(j+l) !]}{(x-y)^{i+j+k+l+2}}+\text { reg. }
\end{aligned}
$$

Observe that, upon the correspondence $\partial_{y}^{n} \frac{1}{x-y} \longleftrightarrow \partial_{y}^{n} \delta(x-y)$, the OPE (1.17) corresponds to the PB (1.10), with a quantum correction given by the last $\mathcal{O}\left(\hbar^{2}\right)$ term. In particular, noting that $\hat{T}=\frac{1}{2} \hat{W}_{1,0}$, we can confirm that the central charge of our system is $c=-1$, which is a special case of a well-known result about $\beta$ - $\gamma$ systems.

We now come to the main point, namely, the implication of the normal ordered version of the syzygy (1.7). A straightforward calculation, based on the normal ordering rearrangement identities given, for instance, in [14], leads to the following result:

$$
\begin{aligned}
& \left(\hat{W}_{i, j} \hat{W}_{k, l}\right)-\left(\hat{W}_{i, k} \hat{W}_{j, l}\right)+\left(\hat{W}_{i, l} \hat{W}_{j, k}\right)= \\
& \hbar\left[C_{i, k, l}^{j} \hat{W}_{j, i+k+l+1}+C_{i, l, j}^{k} \hat{W}_{k, i+j+l+1}+C_{i, j, k}^{l} \hat{W}_{l, i+j+k+1}\right],
\end{aligned}
$$


with linear combination coefficients given by

$$
C_{i, j, k}^{l}=\left[\frac{\left((-1)^{k}+(-1)^{j+1}\right)(j+k) !}{(j+k+1) !}+\frac{(-1)^{i}(i+k) !}{(i+k+1) !}+\frac{(-1)^{i+1}(i+j) !}{(i+j+1) !}\right] .
$$

Similarly to the classical case, by using only $\partial \hat{W}_{a, b}=\hat{W}_{a+1, b}+\hat{W}_{a, b+1}$ we can express the generators $\left\{\hat{W}_{i, j} \mid \forall i, j\right\}$ in terms of a subset of the generators spanning one dimensional subspaces of $\hat{\mathcal{V}}_{2 r}$,

$$
\hat{\mathcal{V}}_{d}:=\text { linear } \operatorname{span}\left\{\hat{W}_{i, j} \mid i+j+1=d\right\},
$$

for $r=1,2, \ldots$ any natural number. However, since the r.h.s. of (1.18a) is nonzero for $\hbar \neq 0$, it is clear that using also $(1.18 \mathrm{a})$ we can now eliminate the remaining generators lying in $\hat{\mathcal{V}}_{2 r}$ as well if $2 r$ can be written in the form $2 r=i+j+k+l+2$ with pairwise distinct nonnegative integers $i, j, k, l$. But this is always possible if $2 r \geq 0+1+2+3+2=8$, which implies that the commutant $\hat{\mathcal{P}}_{s l(2)}$ is generated by (repeated) derivatives and normal ordered products of the following finite set of fields:

$$
\hat{T}=\frac{1}{2} \hat{W}_{1,0}, \quad \hat{W}_{1,2}, \quad \hat{W}_{1,4},
$$

having scale dimensions $2,4,6$. This is a drastic difference from the classical case where we need infinitely many generating fields since the r.h.s. of the syzygy (1.7) is zero in the classical case, when $\hbar=0$. The (automatically closed, nonlinear) OPE algebra of the fields (1.20) can be found from (1.17). It is also easy to find a generating set of the commutant $\hat{\mathcal{P}}_{\text {sl(2) }}$ consisting of $\hat{T}$ and primary fields with weights 4 and 6 . In conclusion, the above construction yields a quantum $\mathcal{W}(2,4,6)$ algebra at $c=-1$ possessing an infinitely, nonfreely generated classical limit. Next we shall identify this $\mathcal{W}$-algebra as the coset of $\widehat{s l(2)}$ at level $-\frac{1}{2}$ with respect to the horizontal subalgebra.

In order to establish the isomorphism given by the second equality in

$$
\hat{\mathcal{P}}_{s l(2)}:=\frac{\hat{\mathcal{P}}}{s l(2)}=\frac{\widehat{s l(2)}-\frac{1}{2}}{s l(2)}
$$

where $\widehat{s l(2)}{ }_{-\frac{1}{2}} / \operatorname{sl}(2)$ denotes the commutant of the horizontal $\operatorname{sl}(2)$ in $\widehat{s l(2)}{ }_{-\frac{1}{2}} \subset \hat{\mathcal{P}}$, it is enough to verify that the generators of $\hat{\mathcal{P}}_{s l(2)}$ given by $(1.20)$ can be expressed in terms of the $\widehat{s l(2)}{ }_{-\frac{1}{2}}$ currents given by (1.13). The quantum Sugawara Virasoro of the current algebra is given by

$$
3 \hbar \hat{T}_{\mathrm{sug}}=(\hat{J} \cdot \hat{J})=\frac{1}{2}\left(\hat{J}_{H} \hat{J}_{H}\right)+\left(\hat{J}_{E} \hat{J}_{F}\right)+\left(\hat{J}_{F} \hat{J}_{E}\right) .
$$

Using the rearrangement identities of [14], one can prove the equality

$$
\hat{T}_{\text {sug }}=\hat{T}
$$


where $\hat{T}$ is given by (1.12). Note that the l.h.s. of (1.23) is quartic in the basic fields $\hat{\xi}_{ \pm}$, while the r.h.s. is quadratic (reminding us of the "symmetric space theorem" [15]). Clearly, such an equality is only possible at the quantum level, and in fact the classical analogue of the Sugawara expression (1.22) vanishes identically.

Concerning the weight 4 and the weight 6 generators, we can establish the following identities:

$$
(\partial \hat{J} \cdot \partial \hat{J})=\frac{1}{2}\left(\hat{W}_{1,0} \hat{W}_{1,0}\right)-\hbar\left[\frac{1}{6} \partial^{2} \hat{W}_{1,0}+\frac{5}{3} \hat{W}_{1,2}\right]
$$

and

$$
\left(\partial^{2} \hat{J} \cdot \partial^{2} \hat{J}\right)=\frac{1}{2}\left(\partial \hat{W}_{1,0} \partial \hat{W}_{1,0}\right)-2\left(\hat{W}_{1,0} \hat{W}_{1,2}\right)-\hbar\left[\frac{1}{60} \partial^{4} \hat{W}_{1,0}+\frac{38}{15} \partial^{2} \hat{W}_{1,2}-\frac{17}{5} W_{1,4}\right]
$$

It is easy to see that using the above identities one can express the generating set (1.20) of $\hat{\mathcal{P}}_{s l(2)}$ in terms of composites of the current $\hat{J}$. (Observe that this is possible because quantum corrections are present on the r.h.s. of (1.24) and (1.25)). This proves the relation

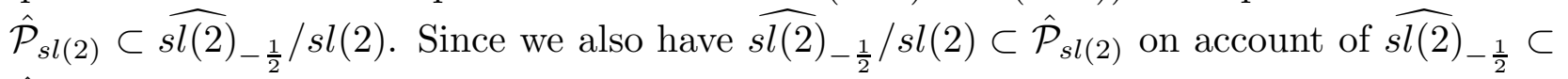
$\hat{\mathcal{P}}$, by (1.13), the isomorphism (1.21) has been now established.

\section{The deformable $\mathcal{W}(2,4,6)$ and remarks on diagonal cosets}

In this section we explain the origin of the 'fourth' deformable $\mathcal{W}(2,4,6)$-algebra, which was unexpectedly found in [6] in addition to the 3 expected deformable algebras with the same weights (the DS type $\mathcal{W}_{\mathcal{S}}^{\mathcal{G}}$-algebras — or 'Casimir algebras' - corresponding to the principal $s l(2)$ embedding in $B_{3}, C_{3}$ and the bosonic projection of the $N=1$ super Virasoro algebra). The explanation will be given in terms of a coset construction. We also present related general considerations on coset constructions of $\mathcal{W}$-algebras, arguing that in the classical case the diagonal cosets generically yield infinitely, nonfreely generated algebras.

One can calculate the vacuum character, $\chi_{0}(q)$, of the $\mathcal{W}(2,4,6)$ constructed in the previous section at $c=-1$ from either of its coset realizations in (1.21). One obtains (see also [16]) the following formula:

$$
\chi_{0}(q)=\frac{\varphi_{0}(q)-q \varphi_{2}(q)}{\prod_{n>0}\left(1-q^{n}\right)^{2}} \quad \text { with } \quad \varphi_{n}(q):=\sum_{m \geq 0}(-1)^{m} q^{\frac{m(m+1)}{2}+m n}
$$

If one compares this with the standard 'vacuum Verma module character' $\phi_{2,4,6}(q)$ associated to fields with weights $2,4,6$, given by

$$
\phi_{2,4,6}(q):=\frac{1}{\prod_{n \geq 0}\left(1-q^{n+2}\right)\left(1-q^{n+4}\right)\left(1-q^{n+6}\right)}
$$


one finds up to order 20

$\chi_{0}(q)-\phi_{2,4,6}(q)=-q^{11}\left(1+2 q+3 q^{2}+6 q^{3}+10 q^{4}+17 q^{5}+27 q^{6}+44 q^{7}+67 q^{8}+105 q^{9}+\mathcal{O}\left(q^{10}\right)\right)$.

The fact that all coefficients on the r.h.s. of (2.3) are nonpositive and $\phi_{2,4,6}(q)$ is the smallest standard character for which this is the case not only confirms (by the argument of $[17,1]$ ) that the coset $(1.21)$ should be a $\mathcal{W}(2,4,6)$-algebra, what we have already proved, but also shows the existence of a first 'null field' at scale dimension 11. The existence of this null field is due to the normal ordered syzygy (1.18). Indeed, at scale dimension 11 eq. (1.18a) yields 3 relations, correspondingly to the 3 different possible choices of pairwise distinct $i, j, k, l$ such that $i+j+k+l+2=11$. Two linear combinations of these relations arise as derivatives of the relations at scale dimensions 8 and 10 which were used to express the scale dimension 8 and 10 'would-be-generators' ( $\hat{W}_{1,6}$ and $\left.\hat{W}_{1,8}\right)$ in terms of the set $(1.20)$. The remaining third relation gives rise to the null field in question. Along these lines, it is also easy to derive the explicit formula of this vanishing nontrivial normal ordered differential polynomial in the generators (1.20), but the formula is not particularly enlightening. What is important is to emphasize the twofold rôle of the normal ordered relations: A subset of them is responsible for the algebra being finitely generated at the quantum level and the rest give rise to null fields.

Let us recall now that it was shown in [7] that the so far unexplained algebra with spins 2, 4 and 6 has a generic null field precisely at this scale dimension. It is also known that the bosonic projection of the $N=1$ super Virasoro algebra has a generic null field already at scale dimension 10 [17], whereas the Casimir algebras of $B_{3}, C_{3}$ have no null fields at $c=-1$. In conclusion, we can identify the coset algebra (1.21) as the 'fourth' deformable $\mathcal{W}(2,4,6)$-algebra at $c=-1$. In order to understand how to deform this coset algebra to generic $c$ and thus completely explain the algebra, let us proceed with some general remarks on cosets.

Consider a coset of the form $\left(\widehat{\mathcal{G}}_{k} \oplus \widehat{\mathcal{G}}_{m}\right) / \widehat{\mathcal{G}}_{k+m}$, where $\mathcal{G}$ is a simple Lie algebra, $\widehat{\mathcal{G}}_{k}$ is the corresponding affine Kac-Moody algebra at level $k$, and $\widehat{\mathcal{G}}_{k+m} \subset \widehat{\mathcal{G}}_{k} \oplus \widehat{\mathcal{G}}_{m}$ is the diagonal embedding. In such a situation one can show that

$$
\lim _{m \rightarrow \infty} \frac{\widehat{\mathcal{G}}_{k} \oplus \widehat{\mathcal{G}}_{m}}{\widehat{\mathcal{G}}_{k+m}}=\frac{\widehat{\mathcal{G}}_{k}}{\mathcal{G}},
$$

where the equality holds at the level of algebras. For generic $m$, the coset algebra on the l.h.s. is a deformation of the coset algebra on the r.h.s., in particular, it has the same spin content of generators. This statement holds for classical as well as quantum $\mathcal{W}$-algebras. The argument presented for the quantum case in $[18,19]$ is roughly the following: The coset generators of the 1.h.s. are $\mathcal{G}$-singlets in $\widehat{\mathcal{G}}_{k} \oplus \widehat{\mathcal{G}}_{m}$ with respect to the horizontal subalgebra 
$\mathcal{G} \subset \widehat{\mathcal{G}}_{k+m}$. Denote the currents generating $\widehat{\mathcal{G}}_{k}$ by $J_{a}(a=1, \ldots, \operatorname{dim} \mathcal{G})$, those generating $\widehat{\mathcal{G}}_{m}$ by $j_{a}$. Taking commutators (resp. Poisson brackets) with all $\left(J_{a}+j_{a}\right)$ one concludes from the central terms that the coefficient of a monomial contained in a generator of the coset algebra tends to zero for $m \rightarrow \infty$ if it contains any $j_{a}$. This proves the inclusion of the l.h.s. into the r.h.s of (2.4). The equality in (2.4) as well as deformability follow from the fact that to any $\mathcal{G}$-singlet consisting of the $J_{a}$ only one can add correction terms containing also the $j_{a}$ such that it commutes with $\widehat{\mathcal{G}}_{k+m}[18,19]$.

In the classical case the deformation of the 'singlet algebra' on the r.h.s. of (2.4) can be made explicit as follows. The $\mathcal{G}$-valued current $J$ generating the algebra $\widehat{\mathcal{G}}_{k}$ satisfies the Poisson brackets

$$
\left\{J_{a}(x), J_{b}(y)\right\}=\sum_{c} f_{a b}^{c} J_{c}(y) \delta(x-y)-k g_{a b} \delta^{\prime}(x-y),
$$

where $f_{a b}^{c}\left(g_{a b}\right)$ denote the structure constants (metric) of $\mathcal{G}$, and similarly for the currents $j$ and $(J+j)$ that generate $\widehat{\mathcal{G}}_{m}$ and $\widehat{\mathcal{G}}_{k+m}$, respectively. Let $P=P\left(J, \partial J, \ldots, \partial^{n} J\right)$ be an arbitrary element of the singlet algebra, i.e., a differential polynomial in the components of $J$ which is invariant under the transformation $\delta_{\epsilon} J=[\epsilon, J]$ for any constant $\epsilon \in \mathcal{G}$. The corresponding element of the diagonal coset on the l.h.s. of (2.4) is obtained by replacing $J$ by $I$, where

$$
I:=J-\frac{k}{m} j,
$$

and also replacing $\partial^{i} J$ by $\mathcal{D}^{i} I$, where $\mathcal{D}$ is the covariant derivative defined by

$$
\mathcal{D} I:=\partial I+\frac{1}{k+m}[J+j, I],
$$

for $k, m,(k+m)$ nonzero. Indeed, for any $\mathcal{G}$-valued function $\epsilon(x)$, under the transformation

$$
\delta_{\epsilon} J=[\epsilon, J]-k \epsilon^{\prime}, \quad \delta_{\epsilon} j=[\epsilon, j]-m \epsilon^{\prime}
$$

one has

$$
\delta_{\epsilon}\left(\mathcal{D}^{i} I\right)=\left[\epsilon, \mathcal{D}^{i} I\right] .
$$

This immediately implies that $P\left(I, \mathcal{D} I, \ldots, \mathcal{D}^{n} I\right)$ belongs to the coset on the l.h.s. of (2.4), and it tends to $P\left(J, \partial J, \ldots, \partial^{n} J\right)$ as $m \rightarrow \infty$, in accordance with (2.4).

We shall see later in this paper that covariant derivatives are useful also in many other considerations concerning current algebras, simply because the currents generate gauge transformations. See also ref. [9], which inspired some of our considerations.

We now wish to argue that the cosets $\widehat{\mathcal{G}}_{k} / \mathcal{G}$ are classically always infinitely generated with infinitely many relations. To see this we note first that invariant theory $([11,12]$ and 
references therein) applied to the $\mathcal{G}$-invariant differential polynomials in the $\mathcal{G}$-valued current $J$ containing the derivatives $\partial^{n} J$ up to a fixed finite order leads to finitely many generators and a finitely generated set of relations. However, the coset on the r.h.s. of (2.4) consists of the $\mathcal{G}$-invariant differential polynomials with an arbitrary number of derivatives. Thus, in the end, one is looking for invariant polynomials in infinitely many variables, $\partial^{n} J$ for any $n$, and therefore the invariant ring is also generated by infinitely many generators and infinitely many relations. In the spirit of Weyl [11], these would be obtained by inserting the infinitely many variables $\partial^{n} J$ into some finite list of 'typical basic invariants' and a corresponding finite list of 'typical basic relations', which would be given by a first and a second main theorem of invariant theory for the adjoint representation of $\mathcal{G}$ on $\mathcal{G}$. (Unfortunately, we could not find these theorems in the mathematical literature for the adjoint representation in general, but it might be possible to infer the case of the adjoint representation from more general results and constructions of invariant theory). More precisely, since in our context the infinitely many variables $\partial^{n} J$ are linearly related by the action of the derivation $\partial$, using $\partial$ one could always write down additional linear relations between those generators differing only in the places where the derivatives have been inserted (like in (1.8)). However, taking all relations into account one is still left with infinitely many generators (and relations). This argument shows that in the classical case the coset on the r.h.s. of (2.4) is infinitely generated with infinitely many relations. Clearly, the same is true for the diagonal cosets $\left(\widehat{\mathcal{G}}_{k} \oplus \widehat{\mathcal{G}}_{m}\right) / \widehat{\mathcal{G}}_{k+m}$ by deformability.

Let us illustrate the above in the simplest nontrivial case, the classical singlet algebra

$$
\frac{\widehat{s l(2)_{k}}}{s l(2)}
$$

Since in the complex case the adjoint representation of the Lie algebra $s l(2)$ is equivalent to the vector representation of $o(3)$, we can directly apply the results in [11] for describing the generators and relations of the ring of invariant polynomials (2.8). More precisely, we can do this provided we forget about the action of the derivation and, for the moment, consider the variables $\partial^{n} J$ as independent, where $J$ is the $s l(2)$ valued current. According to [11], the invariant polynomials are generated by the quadratic invariants

$$
S(m, n):=\left\langle\partial^{m} J, \partial^{n} J\right\rangle \quad(\forall m, n)
$$

where $\langle\cdot, \cdot\rangle$ is given by the trace in the defining representation of $s l(2)$, and the cubic invariants

$$
S(p, q, r):=\left\langle\left[\partial^{p} J, \partial^{q} J\right], \partial^{r} J\right\rangle \quad(\forall p \neq q \neq r) .
$$

(This is familiar from many physical applications of the rotation group $S O(3)$, where (2.9a) becomes the usual scalar product of two vectors, and (2.9b) the volume of the 
parallelepipedon spanned by three vectors). We now quote the basic relations from [11] (Chapter II.17). Let $\left\{m_{0}, m_{1}, m_{2}, m_{3}\right\}$ be any set of distinct, nonnegative integers, and let $\left\{n_{0}, n_{1}, n_{2}, n_{3}\right\}$ be another set of this type (there could be overlap between the two sets). In addition to the relations expressing the obvious symmetry properties of the invariants (2.9), the basic relations are of the following three types. First,

$$
\operatorname{det}\left[\begin{array}{llll}
S\left(m_{0}, n_{0}\right) & S\left(m_{0}, n_{1}\right) & S\left(m_{0}, n_{2}\right) & S\left(m_{0}, n_{3}\right) \\
S\left(m_{1}, n_{0}\right) & S\left(m_{1}, n_{1}\right) & S\left(m_{1}, n_{2}\right) & S\left(m_{1}, n_{3}\right) \\
S\left(m_{2}, n_{0}\right) & S\left(m_{2}, n_{1}\right) & S\left(m_{2}, n_{2}\right) & S\left(m_{2}, n_{3}\right) \\
S\left(m_{3}, n_{0}\right) & S\left(m_{3}, n_{1}\right) & S\left(m_{3}, n_{2}\right) & S\left(m_{3}, n_{3}\right)
\end{array}\right]=0 .
$$

Second,

$$
S\left(m_{1}, m_{2}, m_{3}\right) S\left(n_{1}, n_{2}, n_{3}\right)+2 \operatorname{det}\left[\begin{array}{lll}
S\left(m_{1}, n_{1}\right) & S\left(m_{1}, n_{2}\right) & S\left(m_{1}, n_{3}\right) \\
S\left(m_{2}, n_{1}\right) & S\left(m_{2}, n_{2}\right) & S\left(m_{2}, n_{3}\right) \\
S\left(m_{3}, n_{1}\right) & S\left(m_{3}, n_{2}\right) & S\left(m_{3}, n_{3}\right)
\end{array}\right]=0 .
$$

Third,

$$
\sum_{m} \pm S\left(m_{1}, m_{2}, m_{3}\right) S\left(m_{0}, n\right)=0
$$

where $n$ is arbitrary and the sum is over the (signed) permutations of $\left\{m_{0}, m_{1}, m_{2}, m_{3}\right\}$. These would generate all relations between the invariants if the variables $\partial^{n} J$ were independent. Taking the action of the derivation into account, we also have the linear relations

$$
\partial S(m, n)=S(m+1, n)+S(m, n+1)
$$

and

$$
\partial S(p, q, r)=S(p+1, q, r)+S(p, q+1, r)+S(p, q, r+1),
$$

where the cubic invariant is of course zero if any of its two arguments coincide.

We can use the linear relations in (2.11), which are analogous to (1.8), to introduce a nonredundant (minimal) generating set of the differential ring of singlets (2.8). Let $N_{s}$ be the number of generators at scale dimension $s$ in the minimal generating set. Fom (2.11), we find the (classical) generating function, $f_{\mathrm{cl}}(u):=\sum_{s} N_{s} u^{s}$, to be given by

$f_{\mathrm{cl}}(u)=\frac{\left(u^{6}-u^{5}+u^{2}\right)}{\left(1-u^{2}\right)\left(1-u^{3}\right)}=u^{2}+u^{4}+2 u^{6}+2 u^{8}+u^{9}+2 u^{10}+u^{11}+2 u^{12}+u^{13}+3 u^{14}+\mathcal{O}\left(u^{15}\right)$.

This infinite spectrum of classical generating fields ${ }^{2}$ is to be contrasted with the corresponding quantum case. We recall $[20,1]$ that, according to character arguments, the quantum

2 Eq. (2.9) defines invariants for any $\mathcal{G}$ and one can confirm already from the quadratic invariants that the classical coset (2.4) is always infinitely generated. 
version of the coset (2.8) is expected to yield a finitely generated algebra. Moreover, for generic $k$ the scale dimensions of the quantum generating fields should be those determined by the following (quantum) generating function, $f_{q}(u)$,

$$
f_{q}(u)=u^{2}+u^{4}+2 u^{6}+2 u^{8}+u^{9}+2 u^{10}+u^{12}
$$

The first difference between the classical and quantum generating functions occurs at scale dimension 11, where we have a classical generator but no quantum one in the respective nonredundant generating sets. The explanation lies in the fact that the first classical relation also occurs at precisely this scale dimension, namely, it is given by (2.10c) with $\left\{m_{0}, m_{1}, m_{2}, m_{3}\right\}=\{0,1,2,3\}$ and $n=0$. Given our experience with the $\beta$ - $\gamma$ example, we now expect the following: A redundant, infinite generating set of the quantum coset is obtained by normal ordering the classical generating fields, and for the quantum coset it must be possible to eliminate the 'would-be-generator' at scale dimension 11 due to a quantum correction in the normal ordered version of (2.10c). In a similar fashion, one can also understand the cancellation of one 'would-be-generator' at scale dimension 12 . The statement that the quantum generating function (2.13) is correct, which is supported but not rigorously proved by the character 'argument', is clearly equivalent to this cancellation taking place at all higher scale dimensions ${ }^{3}$.

The coset

$$
\frac{\left.\widehat{s l(2)_{k}} \oplus \widehat{s l(2)}\right)_{m}}{\widehat{s l(2)})_{k+m}}
$$

can now be treated easily: We combine the general remarks on cosets of type (2.4) with the statements on the coset $\widehat{s l(2)})_{k} / s l(2)$. Classically, a generating set for the coset algebra (2.14) can be obtained as follows. In the generators (2.9) one replaces the current $J$ by the current $I$ as given by (2.6a) and substitutes the derivative $\partial$ by the covariant derivative $\mathcal{D}$ (2.6b). The relations in the coset (2.14) are obtained by the same substitutions applied to the relations $(2.10),(2.11)$ in the coset $\widehat{s l(2)})_{k} / \operatorname{sl}(2)$.

The quantum version of the coset (2.14) is more complicated because, due to the covariant derivative $\mathcal{D}$, the generators of the quantum algebra cannot be obtained by naively normal ordering the classical generators. At least, deformability ensures that for generic

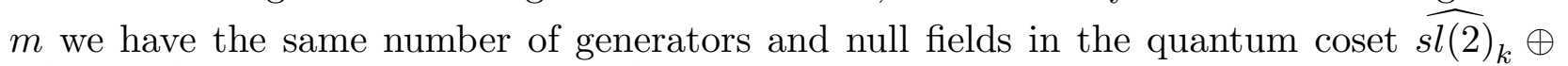
$\widehat{s l(2)_{m}} / \widehat{s l(2)_{k+m}}$ as in the coset $\left.\widehat{s l(2)}\right)_{k} / s l(2)$. In particular, eq. (2.13) means that the quantum coset $(2.14)$ leads to a $\mathcal{W}(2,4,6,6,8,8,9,10,10,12)$ (with known truncations for

3 Similarly to the $\beta-\gamma$ example, only a subset of the relations should be needed for the cancellation, the rest should give rise to generic - in $k$ - null fields. In fact, the first such generic null fields appears at scale dimension 13. 
integer positive $m<6[20,1])$. Still, the results from invariant theory also simplify the explicit construction of the generators of this quantum coset. Noting that $\widehat{s l(2)}$ is generated by the two subalgebras $s l(2)$ and $\widehat{U(1)}$ one may restrict the ansatz for invariant fields to normal ordered differential polynomials in the basic invariants $(2.9)$ where each $s l(2)$ valued current $J$ can either be replaced by $J \in \widehat{s l(2)_{k}}$ or by $j \in \widehat{s l(2)}$. Then, one determines the coefficients in this ansatz by requiring the OPE with the $U(1)$-current in the diagonally embedded $\widehat{s l(2)}$ to be regular. This enables one to explicitly construct at least the primary generator of dimension 4 in the coset (2.14) in addition to the Virasoro field.

Before proceeding with the explanation of the deformable quantum $\mathcal{W}(2,4,6)$, we would like to note a well-known result for the central charge $c$ of the quantum coset (2.14):

$$
c_{k}(m)=\frac{3 k}{k+2}\left(1-\frac{2(k+2)}{(k+m+2)(m+2)}\right) .
$$

Now we return to the starting point of this section and give the explanation of the 'fourth' deformable $\mathcal{W}(2,4,6)$. Recall that we have already identified this algebra at $c=-1$ as the coset $\widehat{s l(2)}{ }_{-\frac{1}{2}} / \operatorname{sl}(2)$. Eq. (2.4) enables us to deform the quantum coset $\widehat{s l(2)}{ }_{-\frac{1}{2}} / \operatorname{sl}(2)$ to generic $c$ (respective $m$ ) in the following manner ${ }^{4}$ :

$$
\frac{\widehat{s l(2)}{ }_{-\frac{1}{2}} \oplus \widehat{s l(2)} m}{\widehat{s l(2)_{m-\frac{1}{2}}}} \cong \mathcal{W}(2,4,6) .
$$

In particular, also the null field at scale dimension 11 is deformed to generic $c$. This shows that the coset $(2.16)$ realizes the previously not understood solution for $\mathcal{W}(2,4,6)$. The relation between the central charge $c$ and the level $m$ is given by $(2.15): c=c_{-\frac{1}{2}}(m)=$ $-\frac{(2 m+7) m}{(2 m+3)(m+2)}$. (We have not assumed $m$ to be an integer and therefore we have indeed constructed the algebra for generic $c$ ). When looking for minimal models of this $\mathcal{W}(2,4,6)$ particular values of the level $m$ will be distinguished. Note that, by an explicit search, a few minimal models of this algebra have been found in [7] for values of $c$ where the level $\mathrm{m}$ is an integer. It would certainly be interesting to find out if this is true in general and to better understand the minimal models of the coset algebra (2.16).

We conclude the discussion of the example (2.16) by remarks on how the truncation happens at $k=-\frac{1}{2}$ in the generically larger coset algebra (2.14). For the quantum coset $\widehat{s l(2)})_{k} / s l(2)$ we have checked that one 'would-be-generator' for each of the scale dimensions 6,8 and 9 becomes a null field at $k=-\frac{1}{2}$. This probably also applies to the quantum analogue of the classical generator at scale dimension 11. Therefore, the first classical relation, which arises at scale dimension 11, is now not needed to cancel a 'would-begenerator', but can give rise to the first null field (the one we already understood in the $\beta-\gamma$

4 This observation is due to R. Blumenhagen. 
realization). By deformability, the same remarks apply to the quantum coset (2.16). The picture in the classical case is less clear. From the quantum equivalence in (1.21) and our study of the classical analogue $\mathcal{P}_{s l(2)}$ of $\hat{\mathcal{P}}_{s l(2)}$, we expect the classical limit of the quantum coset (2.16) to contain one generator for each positive even scale dimension. However, in the classical case nothing happens to the generators at any value of the level $k \neq 0$, and therefore the set of generators of the 'full classical coset' is encoded in the counting function (2.12) also at $k=-\frac{1}{2}$. This apparent contradiction can probably be explained by the existence of a subring closed under Poisson bracket, containing one generator for each $d$, $0<d \in 2 \mathbb{Z}$, in the full classical coset given by the ring of all classical invariants. We expect the classical limit of (2.16) to realize such a proper subring of the full classical coset.

Recall that at $k=1$ the quantum coset (2.14) gives just the Virasoro algebra. The classical limit of this Virasoro field freely generates a subring of the full classical coset, which is obviously closed under the Poisson bracket. (As noted above, at $k=-\frac{1}{2}$ we also expect a subring, but an infinitely generated one, to realize the classical limit). A similar remark applies to the cosets $\widehat{\mathcal{G}}_{1} \oplus \widehat{\mathcal{G}}_{m} / \widehat{\mathcal{G}}_{m+1}$ for $\mathcal{G}$ any simply laced Lie algebra $A$, $D, E$. The quantum versions of these cosets are well-known to give rise to the so-called 'Casimir algebras' (see e.g. [14]) which contain no null fields for generic $m$. On the other hand, at $m=\infty$ the $\operatorname{rank}(\mathcal{G})$ classical Casimir invariants (invariants without derivatives of the current) freely generate a subring of the ring of all classical invariants, which is closed under Poisson bracket [21]. Clearly, the deformation of this subring to generic $m$ carries the classical limit of the quantum Casimir algebra.

\section{On general cosets and the deformable $\mathcal{W}(2,3,4,5)$-algebra}

In this section we give a prescription for finding the generating set for a general class of (classical) cosets, generalizing the results on the diagonal cosets (2.4) discussed previously. In particular, this shows that classical cosets are infinitely, nonfreely generated in general. These results will be illustrated with another example which, as a by-product, also explains the quantum $\mathcal{W}(2,3,4,5)$-algebra found in [8] in addition to the well understood Casimir algebra based on $A_{4}$ that has the same spectrum of generators.

Let $\mathcal{W}$ be any classical $\mathcal{W}$-algebra (including Kac-Moody algebras) generated by finitely many, independent generating fields, and suppose that $\mathcal{W}$ contains a current algebra, $\widehat{\mathcal{G}}_{\kappa}$, as a proper subalgebra. Suppose also that the restriction of the central term of $\mathcal{W}$ to $\widehat{\mathcal{G}}_{\kappa}$ is nondegenerate, and that it is possible to partition the generating fields of $\mathcal{W}$ into the generating fields $J_{a}$ of $\widehat{\mathcal{G}}_{\kappa}$ and a complementary set of generating fields $J_{i}^{\perp}$ that form primary field multiplets with respect to the current algebra $\widehat{\mathcal{G}}_{\kappa}$. (To avoid confusion, note that the $J_{i}^{\perp}$ need not be Kac-Moody currents). Thus the Poisson brackets between the $J_{a}$ are similar to those in (2.5), with a nondegenerate matrix $g_{a b}$, except that now we do not 
require the horizontal subalgebra $\mathcal{G} \subset \widehat{\mathcal{G}}_{\kappa}$ to be (semi) simple — in particular, it can contain $U(1)$ factors. Moreover, we have Poisson brackets of the form

$$
\left\{J_{a}(x), J_{i}^{\perp}(y)\right\}=-\sum_{j} R(a)_{i}^{j} J_{j}^{\perp}(y) \delta(x-y)
$$

where the matrices $R(a)=R(a)_{i}^{j}, a=1, \ldots, \operatorname{dim}(\mathcal{G})$, form a - in general reducible representation of $\mathcal{G}$,

$$
R(a) R(b)-R(b) R(a)=\sum_{c} f_{a b}^{c} R(c)
$$

We are interested in the coset algebra

$$
\frac{\mathcal{W}}{\widehat{\mathcal{G}}_{\kappa}}
$$

i.e., the Poisson bracket algebra carried by the ring of those differential polynomials

$$
p=p\left(J, \partial J, \ldots, \partial^{m} J, J^{\perp}, \partial J^{\perp}, \ldots, \partial^{n} J^{\perp}\right)
$$

that Poisson commute with the charges

$$
Q(\epsilon):=\oint d x \epsilon^{a}(x) J_{a}(x)
$$

for arbitrary test functions $\epsilon^{a}(x)$.

We shall show below that the elements of the coset (3.3) are the $\mathcal{G}$-invariant differential polynomials of the type

$$
P=P\left(J^{\perp}, \mathcal{D} J^{\perp}, \ldots, \mathcal{D}^{n} J^{\perp}\right)
$$

where $\mathcal{D} J^{\perp}$ is the covariant derivative defined by

$$
\mathcal{D} J_{i}^{\perp}:=\partial J_{i}^{\perp}+\frac{1}{\kappa} \sum_{a, b, j} J_{b} g^{b a} R(a)_{i}^{j} J_{j}^{\perp},
$$

with $\sum_{b} g^{a b} g_{b c}=\delta_{a c}$. In particular, the elements of the coset (3.3) can be written as polynomials in $J^{\perp}=\left\{J_{i}^{\perp}\right\}$ and its covariant derivatives. The variables in the argument of the r.h.s. of (3.6) belong to the representation $R(a)$ of $\mathcal{G}$, and $P$ must be invariant under the natural action of $\mathcal{G}$ on its arguments, which means that $P$ must Poisson commute with $Q(\epsilon)$ for constant $\epsilon^{a}$. The significance of this result is that it reduces the problem of describing the ring carrying the classical coset (3.3) to a standard (although not necessarily easy) problem in the invariant theory of the finite dimensional Lie algebra $\mathcal{G}$. Thus, applying the same general reasoning as for the diagonal coset, we see that the differential ring of invariants (3.3) must be infinitely, nonfreely generated in general. 
For the proof we first rewrite the polynomial $p$ in (3.4) as a (uniquely determined) polynomial

$$
P=P\left(J, D J, \ldots, D^{m} J, J^{\perp}, \mathcal{D} J^{\perp}, \ldots, \mathcal{D}^{n} J^{\perp}\right)
$$

in the new variables $\mathcal{D}^{k} J_{i}^{\perp}, D^{k} J_{a}$, where the new derivative $D^{k} J$ of the $\mathcal{G}$-valued current $J$ is recursively defined by

$$
D^{k} J:=\partial D^{k-1} J+\frac{1}{\kappa}\left[J, D^{k-1} J\right] \quad \text { with } \quad D^{0} J=J .
$$

We are looking for $P$ such that

$$
\{Q(\epsilon), P\}=0,
$$

and the new variables are advantageous for computing the Poisson bracket since they have simple Poisson brackets with $Q(\epsilon)$ :

$$
\begin{aligned}
\left\{\left(\mathcal{D}^{k} J^{\perp}\right)_{i}, Q(\epsilon)\right\} & =\sum_{j, a} \epsilon^{a} R(a)_{i}^{j}\left(\mathcal{D}^{k} J^{\perp}\right)_{j} \\
\left\{\left(D^{k} J\right)_{a}, Q(\epsilon)\right\} & =\left[\epsilon,\left(D^{k} J\right)\right]_{a}-\kappa\left(D^{k} \partial \epsilon\right)_{a}
\end{aligned}
$$

where $\epsilon=\left\{\epsilon^{a}\right\}$ is the arbitrary $\mathcal{G}$-valued test function and the derivative $D^{k}(\partial \epsilon)$ is defined similarly to (3.9),

$$
D^{k}(\partial \epsilon):=\partial D^{k-1}(\partial \epsilon)+\frac{1}{\kappa}\left[J, D^{k-1}(\partial \epsilon)\right] \quad \text { with } \quad D^{0}(\partial \epsilon)=\partial \epsilon .
$$

One easily verifies (3.11) by induction on $k$ (or recalls it from Yang-Mills theory). Because of the derivation property of the Poisson bracket, $\{Q(\epsilon), P\}$ is a linear expression in the algebraically independent parameters $\partial^{k} \epsilon^{a}$,

$$
\{Q(\epsilon), P\}=\sum_{a, k} P_{a, k} \partial^{k} \epsilon^{a},
$$

and thus (3.10) requires the polynomials $P_{a, k}$, which depend on the same variables as $P$, to vanish. Let us now single out an index $a$ and look at $P_{a, k_{\max }}$, where $k_{\max }$ is the highest value of $k$ that appears in (3.13) for this particular $a$. It is easy to see from the second line of (3.11) that the contributions to $P_{a, k_{\max }}$ come from those monomials in the polynomial $P$ that contain (various powers of) the highest derivative of the type $\left(D^{l} J\right)_{a}$, given by $\left(D^{k_{\max }-1} J\right)_{a}$, which appears in (3.8). Since those monomials are algebraically independent, $P_{a, k_{\max }}=0$ requires all of them to vanish. This then immediately implies that $P$ in (3.8) cannot depend on the variables $\left(D^{l} J\right)_{a}$ for any $a$ and $l=0,1, \ldots$, proving that $P$ must be of the form (3.6). For such a $P$ the requirement that (3.10) must hold for any test function $\epsilon$ is equivalent to the same requirement for constant $\epsilon$ since no derivative 
of $\epsilon$ appears in the first line of (3.11), completing the proof of our characterization of the elements of the coset (3.3).

It is easy to recover the results on the diagonal coset on the l.h.s. of (2.4) from the above. In that case $\widehat{\mathcal{G}}_{\kappa}, \kappa=k+m$, is the diagonal subalgebra in the denominator and $J^{\perp}$ becomes $I$ in (2.6a). It is also easy to specialize the general result to classical cosets of the type $\widehat{\mathcal{G}}_{k} \oplus \widehat{\mathcal{H}}_{m} / \widehat{\mathcal{H}}_{i k+m}$, where $i$ denotes the Dynkin index of the embedding $\mathcal{H} \subset \mathcal{G}$. The generators comprising $J^{\perp}$ are in this case given by the currents in the complement of $\widehat{\mathcal{H}}_{i k}$ in $\widehat{\mathcal{G}}_{k}$ together with the currents in $\widehat{\mathcal{H}}_{i k} \oplus \widehat{\mathcal{H}}_{m}$ formed according to (2.6a). From this one can derive the natural generalization of (2.4):

$$
\lim _{m \rightarrow \infty} \frac{\widehat{\mathcal{G}}_{k} \oplus \widehat{\mathcal{H}}_{m}}{\widehat{\mathcal{H}}_{i k+m}}=\frac{\widehat{\mathcal{G}}_{k}}{\mathcal{H}} .
$$

It also interesting to note that this type of coset has subalgebras according to

$$
\frac{\widehat{\mathcal{G}}_{k}}{\widehat{\mathcal{H}}_{i k}} \subset \frac{\widehat{\mathcal{G}}_{k} \oplus \widehat{\mathcal{H}}_{m}}{\widehat{\mathcal{H}}_{i k+m}} \supset \frac{\widehat{\mathcal{H}}_{i k} \oplus \widehat{\mathcal{H}}_{m}}{\widehat{\mathcal{H}}_{i k+m}}
$$

and our description applies to the full coset as well as to these subcosets separately. When dealing with the coset $\widehat{\mathcal{G}}_{k} / \widehat{\mathcal{H}}_{i k}$ the variable $J^{\perp}$ becomes the component of the $\mathcal{G}$-valued current generating $\widehat{\mathcal{G}}_{k}$ that lies in the orthogonal complement $\mathcal{H}^{\perp}$ in the decomposition $\mathcal{G}=\mathcal{H} \oplus \mathcal{H}^{\perp}$; thus explaining our notation.

The relations (3.14) and (3.15) are also valid in the quantum case. The r.h.s. of (3.14) can be quantized by just normal ordering, whereas for the quantum analogue of the l.h.s. this holds at least in the limit $\hbar \rightarrow 0$. It follows that for generic values of the parameters there must be a one-to-one linear correspondence between the fields in the classical and respective quantum cosets. (It is the algebraic relations based on the ordinary product and on the normal ordered product which are different in the classical and corresponding quantum case). In particular, this means that for generic parameters the number of invariant fields at a fixed scale dimension in the quantum coset (3.14) is equal to the number of fields in the classical coset. (Clearly, this applies to the general case (3.3) as well). This observation can be utilized when calculating the vacuum character of a quantum coset algebra for generic parameters.

Now we apply the above results to a particular class of classical cosets with $\widehat{\mathcal{G}}_{\kappa}=\widehat{U(1)}$. Consider a $\mathcal{W}$-algebra which can be divided into a $\widehat{U(1)}$ subalgebra and a complement consisting of $\widehat{U(1)}$-primary charge conjugate fields with $U(1)$ charge \pm 1 and charge neutral fields. In this case the polynomials (3.6) which are invariant under global $U(1)$ transformations are the charge neutral combinations. The charge neutral generators of $\mathcal{W}$ simply survive the reduction and therefore we focus our attention on the charged fields. Denote 
the charge conjugate doublets by $W_{a}^{ \pm}$and their covariant derivatives by $W_{a}^{ \pm,(i)}=\mathcal{D}^{i} W_{a}^{ \pm}$. Then a redundant generating set for the coset is given by the $\widehat{U(1)}$-primary charge neutral fields and the following composite generators:

$$
U_{a, b}^{i, j}:=W_{a}^{+,(i)} W_{b}^{-,(j)}
$$

Forgetting about the action of the derivative $\mathcal{D}$, all relations satisfied by the redundant set of generators (3.16) are generated by the following quadratic relations:

$$
U_{a, b}^{i, j} U_{c, d}^{k, l}-\epsilon_{b, c} \epsilon_{b, d} \epsilon_{d, c} U_{a, d}^{i, l} U_{c, b}^{k, j}=0
$$

where the 'statistics factor' $\epsilon_{a, b}$ is defined as $\epsilon_{a, b}=-1$ if both $W_{a}^{ \pm}$and $W_{b}^{ \pm}$are fermions and 1 otherwise. The proof that (3.17) generates all relations between the generators (3.16) is a particularly simple version of a standard argument used in invariant theory (see the straightening algorithm in [13]). One has to show that a basis for the linear space of invariant polynomials in the $W_{a}^{ \pm,(i)}$ with a given degree can be obtained from (3.16) using (3.17) and (anti-)commutativity of the $U_{a, b}^{i, j}$. Because the invariant monomials have zero charge they contain an equal number of $W_{a}^{+,(i)}$ and $W_{a}^{-,(i)}$, in particular they are even order. We can choose an ordering where the charge alternates. This reduces the problem to finding a basis for monomials of type $W_{a}^{+,{ }^{(i)}} W_{b}^{-,{ }^{(j)}} W_{c}^{+,{ }^{(k)}} W_{d}^{-,{ }^{(l)}} \ldots$.. Clearly, a basis for these monomials is obtained by a lexicographic ordering of the sets $\{(a, i),(c, k), \ldots\}$ and $\{(b, j),(d, l), \ldots\}$. Then observe that, on the one hand, each such monomial can be written as a monomial in the generators (3.16). On the other hand, each set of indices can be independently ordered for monomials in the $U_{a, b}^{i, j}$ using (3.17) and (anti-)commutativity of the $U_{a, b}^{i, j}$. This completes the proof.

Finally, we enforce the action of the covariant derivative on the generators (3.16):

$$
\mathcal{D} U_{a, b}^{i, j}=U_{a, b}^{i+1, j}+U_{a, b}^{i, j+1}
$$

Using (3.18) we can eliminate generators from the set (3.16) in favour of a nonredundant set of generators, for example the generators $U_{a, b}^{0, j}$. As usual, the complete set of relations satisfied by the nonredundant generating set $U_{a, b}^{0, j}$ can be obtained from (3.17) using (3.18). (We note in passing that the relation for the coset $W_{3}^{2} / \widehat{U(1)}$ discussed in [9], eq. (2.26) of the reference, can be recovered as a particular consequence of (3.17) and (3.18)).

The general statements will now be illustrated in the following example: The commutant of the $U(1)$-current in the $N=2$ super Virasoro algebra, $\operatorname{SVIR}(N=2)$. The algebra $\operatorname{SVIR}(N=2)$ is generated by the energy momentum tensor $L$, a current $J$ and two fermionic fields $G^{ \pm}$of scale dimension $\frac{3}{2}$ carrying $U(1)$-charge \pm 1 . In order to find the complement of the $\widehat{U(1)}$ current algebra in $\operatorname{SVIR}(N=2)$ we first go to a primary basis with respect to 
the current algebra. This means that we have to replace the energy momentum tensor $L$ by $\hat{L}=L-\frac{3}{2 c} J J$ if we fix the normalization such that the central term of $J$ with itself is given by $\frac{c}{3}$. Next we introduce the covariant derivative according to (3.7):

$$
\begin{aligned}
\mathcal{D} \hat{L} & :=\partial \hat{L}, \\
\mathcal{D} G^{ \pm} & :=\partial G^{ \pm} \pm \frac{3}{c} J G^{ \pm} .
\end{aligned}
$$

From (3.16) and the action (3.18) of the covariant derivative $\mathcal{D}$ we obtain a nonredundant generating set for the classical coset $\operatorname{SVIR}(N=2) / \widehat{U(1)}$ :

$$
\begin{aligned}
\hat{L} & =L-\frac{3}{2 c} J J, \\
\left\{U^{0, j}\right. & \left.=G^{+} \mathcal{D}^{j} G^{-} \mid 0 \leq j \in \mathbb{Z}\right\} .
\end{aligned}
$$

Furthermore, we obtain from (3.17) the complete set of relations (in addition to (3.18)) satisfied by the redundant set of generators $U^{i, j}=\left(\mathcal{D}^{i} G^{+}\right)\left(\mathcal{D}^{j} G^{-}\right)$:

$$
U^{i, j} U^{k, l}+U^{i, l} U^{k, j}=0 .
$$

An important special case of (3.21) for the nonredundant set of generators (3.20b) is

$$
U^{0, j} U^{0, k}=0 \quad \forall j, k .
$$

These relations also directly follow from the fact that $G^{ \pm}$are fermions satisfying the Pauli principle. Note that the nonredundant set of generators (3.20) as well as the set of relations they satisfy are infinite, as expected.

Let us now turn to the quantum version of this coset. For generic $c$, counting the number of invariant fields at a given scale dimension should be the same at the classical and the quantum level. Therefore one can calculate to vacuum character, $\chi_{0}$, by counting the number of classical invariants that arise as differential polynomial in $\hat{L}$ and $G^{ \pm}$. In this way one obtains

$$
\chi_{0}(q)-\phi_{2,3,4,5}(q)=-q^{8}\left(2+4 q+9 q^{2}+16 q^{3}+32 q^{4}+54 q^{5}+96 q^{6}+\mathcal{O}\left(q^{7}\right)\right),
$$

where $\phi_{2,3,4,5}$ denotes the vacuum character of a freely generated algebra with generators of scale dimension 2, 3,4 and 5 . This suggests the identification

$$
\frac{\operatorname{SVIR}(N=2)}{\widehat{U(1)}} \cong \mathcal{W}(2,3,4,5)
$$

for the quantum coset. According to (3.23), our quantum coset has two generic null fields at scale dimension 8 . Recall now that the same null field structure has been observed in $[8]$ for 
the 'second' deformable $\mathcal{W}(2,3,4,5)$-algebra found by direct construction. Therefore the $\mathcal{W}(2,3,4,5)$-algebra appearing in the identification (3.24) must be the 'second' $\mathcal{W}(2,3,4,5)$ algebra of [8] (the other deformable $\mathcal{W}(2,3,4,5)$-algebra is the Casimir algebra of $A_{4}$ which has no generic null field).

The identification (3.24) is further supported by explicit calculations presented in detail in [22]. The energy momentum tensor of the quantum coset is just the normal ordered version of (3.20a). The quantum analogues of the generators of higher scale dimension (3.20b) are more difficult to determine. By commutation with the zero mode of the current it follows that all fields in the coset must be uncharged. It is straightforward to make an ansatz in the uncharged fields and determine those linear combinations that commute with the complete current. One finds precisely one new generator at scale dimensions 3,4 and 5 with leading terms quadratic in the fermions $G^{ \pm}$, and by computing the OPEs of these composite fields one recovers [22] the structure constants of the $\mathcal{W}(2,3,4,5)$ given in [8].

Similarly as in previous examples, one can argue that normal ordered analogues of the classical relations (3.22) ensure that the quantum coset on the 1.h.s. of (3.24) is finitely generated, whereas the corresponding classical coset needs infinitely many generators (3.20). For example, the normal ordered version of the classical relation $G^{+} G^{-} G^{+} G^{-}=0$ contains a term proportional to what would have been the new generator at scale dimension 6 .

The central charge $\hat{c}$ of the coset energy momentum tensor $\hat{L}$ is obtained by shifting the original central charge $c$ by 1 . Inserting the parametrization $c(k)$ of the unitary minimal models of $\operatorname{SVIR}(N=2)$ by a positive integer $k$, one obtains

$$
\hat{c}(k)=c(k)-1=\frac{2(k-1)}{k+2} .
$$

Note that this is just the well-known formula for the central charge of $\mathbb{Z}_{k}$-parafermions [23]. In fact, the coset algebra (3.24) is a universal object [22] for the first unitary minimal models of the Casimir algebras based on $A_{k-1}$ which describe the $\mathbb{Z}_{k}$-parafermions [24,25]. Moreover, it is also well known (see e.g. $[1,26]$ ) that the $\mathbb{Z}_{k}$-parafermions can be realized using the coset $\widehat{s l(2)} / \widehat{U(1)}$, and therefore also this coset algebra should be a universal object for the $\mathbb{Z}_{k}$-parafermions. It can be verified that the standard character counting argument of $[17,1]$ indeed predicts a $\mathcal{W}(2,3,4,5)$ (a finitely generated algebra) for the quantum coset $\widehat{s l(2)} / \widehat{U(1)}$.

Because the above contradicts an earlier claim [10], we wish to draw the reader's attention to the further evidence in [22], which shows that the claim of [10] that the quantum coset $\widehat{s l(2)_{k}} / \widehat{U(1)}$ requires infinitely many generators (one for each integer scale dimension greater than or equal to two) is incorrect as it stands. First, it can be shown [22] that $\widehat{s l(2)})_{k} / \widehat{U(1)}$ is isomorphic to $\operatorname{SVIR}(N=2) / \widehat{U(1)}$, which we have argued here to be finitely 
generated. Second, it can be verified explicitly [22] that the quantum coset $\widehat{s l(2)})_{k} / \widehat{U(1)}$ contains the $\mathcal{W}(2,3,4,5)$ of $[8]$ as subalgebra. Finally, comparing the vacuum character ${ }^{5}$ of this quantum coset, $\chi_{0}$ in (3.23), to the number of composite fields in the $\mathcal{W}(2,3,4,5)$ determined in [8] up to scale dimension 8 , proves that no new generator appears in the coset at scale dimensions 6,7 or 8 , contrary to the claim of [10]. Thus we expect that the coset $\widehat{s l(2)} / \widehat{U(1)}$ is actually isomorphic to the $\mathcal{W}(2,3,4,5)$-algebra with generic null fields constructed in [8], though no complete proof is available at the moment. We hope to present a complete proof along the lines of the $\beta-\gamma$ example and character considerations to arbitrary order elsewhere [16].

\section{Orbifolds of $\mathcal{W}$-algebras}

In this section we show that orbifolds of $\mathcal{W}$-algebras behave very similarly to the cosets discussed in the previous two sections. All known examples of orbifolds are generated finitely at the quantum level, but possess infinitely generated classical analogues with infinitely many relations. One orbifold example that will be discussed here also turns up as the $k=2$ special case of the coset (2.14). Namely, the quantum version of the coset $\left.(\widehat{s l(2)})_{2} \oplus \widehat{s l(2)_{m}}\right) / \widehat{s l(2)_{m+2}}$ leads to a $\mathcal{W}(2,4,6)$-algebra isomorphic to the bosonic projection of the $N=1$ super Virasoro algebra [17]. The structure of this quantum $\mathcal{W}(2,4,6)$ has been investigated in $[17,27,28]$. In particular, the first generic null field appears at scale dimension 10. This bosonic projection is a particular case of the more general 'orbifold'construction on $\mathcal{W}$-algebras.

Any $\mathcal{W}$-algebra (including Kac-Moody algebras) with nontrivial outer automorphisms can be projected onto the invariant subspace under the automorphism group. This so-called 'orbifolding' leads to another $\mathcal{W}$-algebra. For simplicity, we here restrict our attention to $\mathbb{Z}_{2}$ automorphisms $\rho$ that act on the finitely many generators $\left\{W_{a} \mid a \in \mathcal{I} \cup \mathcal{K}\right\}$ as follows:

$$
\begin{array}{ll}
\rho\left(W_{a}\right)=W_{a} \quad \forall a \in \mathcal{K}, \\
\rho\left(W_{b}\right)=-W_{b} \quad \forall b \in \mathcal{I} .
\end{array}
$$

We can divide the index set $\mathcal{I}$ into two subsets: A set $\mathcal{I}_{1}$ referring to bosonic fields and a set $\mathcal{I}_{2}$ referring to fermionic fields transforming nontrivially under the automorphism $\rho$. It is easy to determine a generating set classically. Note that the nontrivial $\rho$-invariant differential polynomials are even order in the $\left\{W_{b} \mid b \in \mathcal{I}\right\}$. Plainly, every even order polynomial can be regarded as a polynomial in quadratic expressions. Therefore the quadratic expressions formed out of the $\left\{W_{b} \mid b \in \mathcal{I}\right\}$ generate the orbifold together with the invariant

5 Note that the corresponding formula (4.14) in [10] contains a misprint: The exponent of $f(q)$ should be 2 , not 3 . 
fields $\left\{W_{a} \mid a \in \mathcal{K}\right\}$. A redundant set of quadratic generators is given by:

$$
X_{b, c}^{i, j}:=W_{b}^{(i)} W_{c}^{(j)} \quad b, c \in \mathcal{I}, 0 \leq i, j \in \mathbb{Z}
$$

where $W_{b}^{(i)}:=\partial^{i} W_{b}$. The derivative acts on the generators (4.2) like in eq. (1.8):

$$
\partial X_{b, c}^{i, j}=X_{b, c}^{i+1, j}+X_{b, c}^{i, j+1} .
$$

Using the action of the derivative (4.3) and paying attention to the Pauli principle for the fermionic generators, i.e., that fermions have odd Grassmann parity, one can choose the following minimal set of generators for the orbifold:

$$
\begin{array}{lll}
W_{a}, a \in \mathcal{K} & \text { (invariant generators) } \\
X_{b, c}^{0, j}:=W_{b} \partial^{j} W_{c}, & b<c, b, c \in \mathcal{I}, 0 \leq j \in \mathbb{Z}, & \\
X_{d, d}^{0, j}:=W_{d} \partial^{j} W_{d}, & d \in \mathcal{I}_{1}, 0 \leq j \in 2 \mathbb{Z} & \text { (square of bosons) } \\
X_{e, e}^{0, j}:=W_{e} \partial^{j} W_{e}, & e \in \mathcal{I}_{2}, 0<j \in 2 \mathbb{Z}+1 & \text { (square of fermions) }
\end{array}
$$

where ' $b<c$ ' denotes some ordering of the original generators. Eq. (4.4) shows that $\mathbb{Z}_{2}$ orbifolds are always infinitely generated at the classical level. Note that a (redundant) set of quantum generators can be obtained just by normal ordering (4.4).

In order to find the complete set of relations we first regard all $W_{a}^{(i)}$ as independent. The complete set of relations satisfied by the redundant set of generators (4.2) is generated by

$$
\begin{aligned}
X_{b, c}^{i, j}-\epsilon_{b, c} X_{c, b}^{j, i} & =0, \\
X_{b, c}^{i, j} X_{d, e}^{k, l}-\epsilon_{c, d} X_{b, d}^{i, k} X_{c, e}^{j, l} & =0,
\end{aligned}
$$

where $\epsilon_{b, c}=-1$ if both $W_{b}$ and $W_{c}$ are fermions, and $\epsilon_{b, c}=1$ otherwise. (Clearly, choosing certain indices in (4.5) equal leads to trivial relations). The proof that (4.5) indeed generate all relations is very similar to the one presented below (3.17) and therefore we omit it.

It is straightforward to derive the relations satisfied by the nonredundant set of generators (4.4) from (4.5). One simply has to recursively apply (4.3) (which encodes the action of the derivative) in order to express the relations (4.5) in terms of the generators (4.4).

Next, we further elaborate some of these relations for two examples and discuss their impact on the quantum case. One of the simplest examples of orbifolds is the bosonic projection of the $N=1$ super Virasoro algebra that we have already mentioned. The $N=1$ super Virasoro algebra is the extension of the Virasoro algebra $L$ by a primary scale dimension $\frac{3}{2}$ fermion $G$. According to (4.4), a nonredundant set of generators for the classical orbifold is

$$
L, \quad \Phi^{n}:=G \partial^{n} G \quad \text { for all odd } n .
$$


In particular, this orbifold has one generator at each positive even scale dimension. Using the notation of (4.4) we have the identification $\Phi^{n}=X^{0, n}$ if we omit the irrelevant lower indices. From (4.5b) one reads off $0=X^{0, j} X^{0, l}+X^{0,0} X^{j, l}=X^{0, j} X^{0, l}$ because $X^{0,0}=0$. In terms of the generators (4.6) these infinitely many relations read

$$
0=\Phi^{n} \Phi^{m}=0 \quad \text { for any } \quad 0<n, m \in 2 \mathbb{Z}+1
$$

In this case the particular subset (4.7) of relations for the nonredundant set of generators can also immediately be inferred from the Pauli-principle: $\Phi^{n} \Phi^{m}=\left(G \partial^{n} G\right)\left(G \partial^{m} G\right)=$ $-G^{2}\left(\partial^{n} G\right)\left(\partial^{m} G\right)=0$. However, (4.5) encodes more relations. For example (4.5) and (4.3) imply $X^{0,1} \partial^{2} X^{0,1}=X^{0,1}\left(X^{2,1}+2 X^{1,2}+X^{0,3}\right)=X^{0,1} X^{1,2}=-X^{0,1} X^{1,2}$. For the nonredundant set of generators this implies the following relation at scale dimension 10 :

$$
\Phi^{1} \partial^{2} \Phi^{1}=0
$$

It is also straightforward to verify (4.8) directly: $\Phi^{1} \partial^{2} \Phi^{1}=(G \partial G) \partial^{2}(G \partial G)=$ $G(\partial G)\left(\partial^{2} G\right)(\partial G)+2 G(\partial G)^{2}\left(\partial^{2} G\right)+G(\partial G) G\left(\partial^{3} G\right)=0$. The first and second term on the r.h.s. vanish because $(\partial G)^{2}=0$, the last term is zero because $G^{2}=0$.

Now we explain how the classical relations make the quantum version of the bosonic projection of the $N=1$ super Virasoro algebra generated by just three fields of scale dimension 2, 4 and 6. First, one checks that the normal ordered analogue of the classical relation $\Phi^{1} \Phi^{1}=0$ contains a correction term proportional to $\Phi^{5}$ (for the explicit expression see (A.2) in appendix A). Thus, the field $\Phi^{5}$ with scale dimension 8 does not give rise to a new generator in the quantum case and there is no relation at scale dimension 8 in the quantum orbifold. Similarly, the normal ordered counterpart of the classical relation $\Phi^{3} \Phi^{1}=0$ picks up correction terms containing $\Phi^{7}$ (eq. (A.3) in appendix A). This shows that also at scale dimension 10, upon normal ordering, a classical relation cancels a 'wouldbe-generator'. It follows from general results on $\mathcal{W}$-algebras that this already ensures that the quantum orbifold under discussion at least contains a $\mathcal{W}(2,4,6)$ as subalgebra. We have not yet used the classical relation (4.8). Indeed, on the quantum level there is a generic null field at scale dimension 10, which is the normal ordered counterpart of this classical relation (see eq. (A.4) in appendix A for an explicit formula).

So far one might have the impression that relations arise in orbifolds just because of the Pauli principle. Note that (4.5) also encodes infinitely many relations for orbifolds of bosonic algebras. In order to illustrate these relations and their impact also in the purely bosonic case we briefly comment on a bosonic example. Consider two commuting copies of the Virasoro algebra $\left(L_{1}\right.$ and $\left.L_{2}\right)$ with equal central charge. Then $W:=L_{1}-L_{2}$ is primary with respect to $L:=L_{1}+L_{2}$. Furthermore, $\rho(L)=L$ and $\rho(W)=-W$ is 
an automorphism of this $\mathcal{W}(2,2)$. According to (4.4) the subspace invariant under $\rho$ is generated, in the classical case, by the following fields:

$$
L, \quad \tilde{\Phi}^{n}:=W \partial^{n} W \quad \text { for all even } n
$$

Again, we obtain one generator at each positive even scale dimension. In this case rewriting the relations (4.5) for the redundant set (4.3) in terms of the nonredundant set (4.4) is slightly more complicated. Using the notation $X^{i, j}=W^{(i)} W^{(j)}$ one checks that from (4.3) and (4.5a) $\partial^{2}\left(X^{0,0} X^{0,0}\right)=8 X^{0,1} X^{0,1}+2 X^{0,0} \partial^{2} X^{0,0}$ and $\partial^{2} X^{0,0}=2 X^{1,1}+2 X^{0,2}$. Using (4.5b) it is straightforward to check that $\partial^{2}\left(X^{0,0} X^{0,0}\right)+6 X^{0,0} \partial^{2} X^{0,0}+8 X^{0,0} X^{0,2}=0$. This relation arises at scale dimension 10 , which is the lowest scale dimension admitting a relation. In terms of the generators (4.9) it reads

$$
\partial^{2}\left(\tilde{\Phi}^{0} \tilde{\Phi}^{0}\right)-6 \tilde{\Phi}^{0} \partial^{2} \tilde{\Phi}^{0}+8 \tilde{\Phi}^{0} \tilde{\Phi}^{2}=0
$$

Turning now to the quantum case one can check that the normal ordered counterpart of (4.10) is not identically zero, but contains correction terms including the scale dimension 10 field $\tilde{\Phi}^{6}$ (the explicit formula is eq. (A.6) in appendix A). This indicates that the $\mathbb{Z}_{2}$ orbifold of the quantum $\mathcal{W}(2,2)$ is a $\mathcal{W}(2,4,6,8)$ - a finitely generated algebra, which can be confirmed by an inspection of its vacuum character (see appendix A).

\section{A classical first class Hamiltonian reduction}

In order to convince the reader that at the classical level infinitely, nonfreely generated algebras generically arise in all reduction procedures applied to finitely, freely generated algebras, here we present an example of a first class Hamiltonian reduction where this is the case. Our starting algebra will be the DS type $\mathcal{W}_{\mathcal{S}}^{\mathcal{G}}$-algebra (see e.g. [4]) belonging to the $s l(2)$ embedding $\mathcal{S}$ associated to the long root of $\mathcal{G}=B_{2}$. We next describe the structure of this $\mathcal{W}_{\mathcal{S}}^{\mathcal{G}}$-algebra.

The root diagram of the Lie algebra $\mathcal{G}=B_{2}$ consists of the vectors $\pm e_{1}, \pm e_{2}, \pm\left(e_{1} \pm e_{2}\right)$, and the algebra is spanned by the Cartan-Weyl basis

$$
E_{ \pm e_{1}}, \quad E_{ \pm e_{2}}, \quad E_{ \pm\left(e_{1} \pm e_{2}\right)}, \quad H_{e_{1}}, \quad H_{e_{2}},
$$

normalized by $\left[H_{e_{i}}, E_{e_{i}}\right]=E_{e_{i}}$. We consider the $\operatorname{sl}(2)$ subalgebra $\mathcal{S}=\operatorname{span}\left\{M_{-}, M_{0}, M_{+}\right\}$ belonging to the long root $\left(e_{1}+e_{2}\right)$,

$$
M_{ \pm}:=E_{ \pm\left(e_{1}+e_{2}\right)}, \quad M_{0}:=\frac{1}{2}\left(H_{e_{1}}+H_{e_{2}}\right)
$$


The adjoint representation of $B_{2}$ decomposes under $\mathcal{S}$ as $10=3 \times 1+2 \times 2+3$ and the generating fields of the $\mathcal{W}_{\mathcal{S}}^{\mathcal{G}}$-algebra are the components of the 'highest weight gauge' current, $j_{\mathrm{hw}}(x) \in \operatorname{Ker}\left(\operatorname{ad}_{M_{+}}\right)$, parametrized as

$$
\begin{aligned}
j_{\mathrm{hw}}(x)= & I_{+}(z) E_{-\left(e_{1}-e_{2}\right)}+I_{0}(x)\left(H_{e_{1}}-H_{e_{2}}\right)+I_{-}(x) E_{e_{1}-e_{2}} \\
& +\frac{1}{2} Z_{+}(x) E_{e_{2}}+\frac{1}{2} Z_{-}(z) E_{e_{1}}+\mathcal{L}(x) M_{+} .
\end{aligned}
$$

The fields $I_{0, \pm}$ form an $\widehat{s l(2)}$ Kac-Moody subalgebra of the $\mathcal{W}_{\mathcal{S}}^{\mathcal{G}}$-algebra,

$$
\begin{aligned}
\left\{I_{0}(x), I_{ \pm}(y)\right\} & = \pm I_{ \pm}(y) \delta(x-y) \\
\left\{I_{0}(x), I_{0}(y)\right\} & =\frac{1}{2} \kappa \delta^{\prime}(x-y), \\
\left\{I_{+}(x), I_{-}(y)\right\} & =2 I_{0}(x) \delta(x-y)+\kappa \delta^{\prime}(x-y),
\end{aligned}
$$

where $\kappa$ is a nonzero constant. The fields $Z_{ \pm}$are bosonic fields with conformal weight $\frac{3}{2}$ with respect to the Virasoro $L:=\frac{1}{\kappa}\left(\mathcal{L}+I_{-} I_{+}+I_{0}^{2}\right)$, and form a doublet under the $\widehat{\operatorname{sl}(2)}$ Kac-Moody subalgebra; in particular they have $I_{0}$-charge $\pm \frac{1}{2}$. This fixes almost all the Poisson brackets. The Poisson brackets of $Z_{ \pm}$read

$$
\begin{aligned}
& \left\{Z_{ \pm}(x), Z_{ \pm}(y)\right\}= \pm 2 \kappa\left[I_{ \pm}^{\prime}(y) \delta-2 I_{ \pm}(y) \delta^{\prime}\right] \\
& \left\{Z_{-}(x), Z_{+}(y)\right\}=2\left[\mathcal{L}-\left(I_{-} I_{+}+I_{0}^{2}+\kappa I_{0}^{\prime}\right)\right](y) \delta+4 \kappa I_{0}(y) \delta^{\prime}-2 \kappa^{2} \delta^{\prime \prime}
\end{aligned}
$$

We are interested in the classical Hamiltonian reduction of this $\mathcal{W}_{\mathcal{S}}^{\mathcal{G}}$-algebra defined by the first class constraint

$$
I_{+}(x)=0
$$

The gauge group generated by this constraint acts according to

$$
\begin{aligned}
& L \longrightarrow L, \\
& I_{0} \longrightarrow I_{0}, \\
& Z_{+} \longrightarrow Z_{+}, \\
& Z_{-} \longrightarrow Z_{-}-\epsilon Z_{+}, \\
& I_{-} \longrightarrow I_{-}-2 \epsilon I_{0}+\kappa \epsilon^{\prime},
\end{aligned}
$$

where $\epsilon(x)$ is arbitrary. The problem is just to describe the ring $\mathcal{R}$ of those differential polynomials in the basic fields $L, I_{0}, Z_{+}, Z_{-}, I_{-}$which are invariant under the transformation rule $(5.7)$.

By naive counting we expect that the reduced system should have 4 'functional degrees of freedom'. We have the 3 invariant fields $L, I_{0}, Z_{+}$and can easily construct a fourth 
invariant with the aid of the rational gauge fixing implemented by putting $\epsilon=\frac{Z_{-}}{Z_{+}}$in $(5.7)$, whereby

$$
I_{-} \longrightarrow R=\frac{B}{Z_{+}^{2}} \quad \text { with } \quad B:=Z_{+}^{2} I_{-}-2 Z_{+} Z_{-} I_{0}+\kappa\left(Z_{-}^{\prime} Z_{+}-Z_{-} Z_{+}^{\prime}\right)
$$

The scale dimension 4 differential polynomial $B$ is gauge invariant. Why is our problem not completely trivial? If we were looking for the differential rational gauge invariants then $\left\{I_{0}, Z_{+}, L, R\right\}$ would clearly be a free generating set. However, since it is impossible to make sense of quantum analogues of rational invariants, we are interested in the differential polynomial invariants, and, perhaps contrary to a naive expectation, the set $\left\{I_{0}, Z_{+}, L, B\right\}$ is not a generating set for $\mathcal{R}$. For example, the scale dimension 6 invariant $K \in \mathcal{R}$ given by

$$
K:=\frac{\left(\kappa B^{\prime} Z_{+}^{\prime}-\kappa B Z_{+}^{\prime \prime}-2 B I_{0} Z_{+}^{\prime}\right)}{Z_{+}}
$$

cannot be expressed as a differential polynomial in the set $\left\{I_{0}, Z_{+}, L, B\right\}$, although it can be checked to be a differential polynomial in the basic fields.

Observe also that if we include $K$ into the generating set of $\mathcal{R}$ then it will contain the 5 element subset $\left\{I_{0}, Z_{+}, L, B, K\right\}$ subject to the differential-algebraic relation

$$
Z_{+} K-\kappa B^{\prime} Z_{+}^{\prime}+\kappa B Z_{+}^{\prime \prime}+2 B I_{0} Z_{+}^{\prime}=0
$$

In a sense the problem is to find the higher scale dimension analogues of the invariant $K$ (5.9) and the relation (5.10).

It can be shown (for a proof, see appendix B) that the ring $\mathcal{R}$ is generated by the following gauge invariant differential polynomials:

$$
L, \quad I_{0}, \quad Z_{+}, \quad P_{i, j}
$$

where, for arbitrary nonnegative integers $i, j$,

$$
P_{i, j}=\partial^{i} Z_{+} \mathcal{D}^{j} Z_{-}-\partial^{j} Z_{+} \mathcal{D}^{i} Z_{-}+\frac{1}{\kappa} \sum_{a>0} \mathcal{D}^{a-1} I_{-}\left(\partial^{i} Z_{+} \partial^{j-a} Z_{+}\left(\begin{array}{c}
j \\
a
\end{array}\right)-\partial^{i-a} Z_{+} \partial^{j} Z_{+}\left(\begin{array}{c}
i \\
a
\end{array}\right)\right)
$$

and $\mathcal{D}:=\partial-\frac{2}{\kappa} I_{0}$ is a covariant derivative. These generators of $\mathcal{R}$ are of course not independent. They obey the fundamental nonlinear relations given by

$$
\begin{gathered}
P_{i, j} P_{k, l}-P_{i, k} P_{j, l}+P_{i, l} P_{j, k}=0, \\
P_{i, j} \partial^{k} Z_{+}-P_{i, k} \partial^{j} Z_{+}+P_{j, k} \partial^{i} Z_{+}=0,
\end{gathered}
$$


where $i, j, k, l$ are arbitrary nonnegative integers, and the linear relations given by

$$
\begin{aligned}
P_{i, j} & =-P_{j, i}, \\
\mathcal{D} P_{i, j} & =P_{i, j+1}+P_{i+1, j} .
\end{aligned}
$$

The reader may observe that (5.13-14) are similar to the relations (1.6-8), the reason for this is contained in construction given in appendix B.

The generating set (5.11) is redundant on account of the linear relations (5.14), and using (5.14) like in section 1 we can find a minimal generating set, for instance by keeping only the generators $P_{1,2 s}$ out of the $P_{i, j}$. However, it is unavoidable that the minimal generating set of $\mathcal{R}$ consists of infinitely many generators subject to infinitely many relations. For completeness, we also note that the relation given by $(5.10)$ is recovered from $(5.13 \mathrm{~b})$ by taking $i=0, j=1, k=2$ and observing that $B$ in (5.8) is just $-\kappa P_{1,0}, K$ in (5.9) is proportional to $P_{1,2}$, and $P_{2,0}=\mathcal{D} P_{1,0}$.

We also wish to remark that the reductions related to the so-called $W_{2 n}^{2}$-algebras, which have been partially treated in the appendix of [4], are very similar to the above example; the invariant ring is infinitely generated in those cases, too. The $W_{2 n}^{2}$-cases and the above example are covered by the more general model when one takes a Kac-Moody or $\mathcal{W}$-algebra that contains an $\widehat{s l(2)}$ Kac-Moody subalgebra and imposes a first class constraint of the type (5.6). We hope to further analyze this class of classical reductions and their quantum analogues in a future publication.

\section{Discussion}

In this paper we pointed out a new class of finitely (but nonfreely) generated deformable quantum $\mathcal{W}$-algebras, which consists of the algebras possessing infinitely generated classical limits obeying infinitely many differential-algebraic relations. The existence of this class of $\mathcal{W}$-algebras has not been noted previously, although part of the results needed was already known in the literature.

Our main observation, derived from the examples, is that the infnitely many relations satisfied by the classical generators have the following twofold impact on the corresponding quantum $\mathcal{W}$-algebra. First, using a proper subset of the normal ordered relations one can eliminate the infinitely many 'would-be-generators' in favour of a finite set of quantum generating fields. Second, the generating fields in this finite set are still not independent due to the rest of the normal ordered relations that give rise to generic null fields.

These generic null fields are nontrivial normal ordered differential polynomials in the generating fields that exist for generic $c$ and vanish identically on the defining vacuum representation of the quantum $\mathcal{W}$-algebra. As a terminological aside, we propose to say that 
a finitely generated deformable quantum $\mathcal{W}$-algebra is nonfreely generated if such generic null fields are present.

To be precise, we fully demonstrated the above mentioned statements only in the $\beta$ $\gamma$ example. In the other examples we treated the first few relations and confirmed by character countings that the pairwise cancellation of classical generators against classical relations should happen upon normal ordering also at higher orders. It is a very interesting open question whether this mechanism works to all orders in every case, or - if not - what are the conditions? At least to our knowledge, no example (including $\widehat{s l(2)} / \widehat{U(1)}-$ see section 3) is known for a quantum coset (or other reduced algebra) obtained from a finitely generated algebra for which infinitely many generators are required to describe the reduced quantum $\mathcal{W}$-algebra.

Invariant theory ([11-13]) played an important rôle in our considerations. That this theory should be relevant for $\mathcal{W}$-algebras derives from the fact that, indeed, all known deformable $\mathcal{W}$-algebras can be obtained by reducing simple free field like linear systems or affine Kac-Moody algebras, and in reductions one is always interested in the invariants. The class of invariants most often considered in invariant theory is the polynomial class and in our context the relevant class of invariants is the differential polynomial class, which is not very different. Since, as we have seen throughout the paper, the generators and relations of the differential ring of classical invariants arising in a reduction know a lot about the generators and generic null fields of the corresponding quantum $\mathcal{W}$-algebra, a more extensive application of invariant theory to $\mathcal{W}$-algebras should be a fruitful undertaking.

In this paper we emphasized that in a generic situation the classical coset, orbifold and first class reduction procedures yield reduced classical systems carried by infinitely, nonfreely generated differential rings of invariants. (Incidentally, as far as we know, the classical coset and orbifold reductions lead to infinitely, nonfreely generated classical systems without any nontrivial exception). To avoid confusion, we should also stress that this does not exclude the existence of a (possibly finitely generated) subring closed under Poisson bracket in such a way that only the subring is recovered from the classical limit of a quantum $\mathcal{W}$ algebra resulting from the corresponding quantum reduction procedure. In particular, if the quantum reduction yields a $\mathcal{W}$-algebra which is generated finitely without generic null fields, this is expected to be the case. For instance, this happens for the coset realization of 'Casimir algebras' ( $\mathcal{W}$-algebras in the DS class for principal embeddings), which possess finitely, freely generated classical limits (automatically obtained from classical DS reduction). This provides us with an example where in the classical limit of a quantum $\mathcal{W}$-algebra resulting from a quantum (coset) reduction one recovers only a finitely generated subring of the full ring of invariants resulting from the analogous classical (coset) reduction.

We wish to mention that in [29] DS type classical $\mathcal{W}$-algebras based on $B_{n}$ and $C_{n}$ 
have been recovered from invariant subspaces of those based on some $A_{k}$ by 'folding'. In our sense of 'orbifolding' these subspaces are generated by the invariant generators according to eq. (4.4), and thus represent only a proper subring of the classical orbifold. It should also be noted that the quantum orbifolds of the Casimir algebras based on $A_{k}$ in general have no subalgebras corresponding to Casimir algebras based on a different simple Lie algebra $[22]$.

Finally, we wish to point out that although the finitely but nonfreely generated class of deformable quantum $\mathcal{W}$-algebras considered in this paper seems to be more complicated than the already reasonably well understood class of $\mathcal{W}$-algebras obtained from DS type reductions, it is also relevant for 'physical' applications. For instance, a universal object for the $\mathbb{Z}_{k}$ parafermions (which have been widely used in the literature — see e.g. $[1,23,26]$ ) belongs to this class. Furthermore, in some cases one might want not to include any fermions into the symmetry algebra (for example because they are not observable, or because - from a mathematical point of view - purely bosonic symmetry algebras are sometimes easier to handle), which automatically leads to orbifolds belonging to this new class. The orbifolds obtained from bosonic projection are the chiral algebras of GSO [30] projected models that occur in superstring theory.

\section{Acknowledgements}

We wish to thank R. Blumenhagen, W. Eholzer, K. Hornfeck, R. Hübel, M. Rösgen, W. Nahm, L. O'Raifeartaigh and I. Tsutsui for discussions and for reading the manuscript. We are also grateful to P. Bouwknegt, A. Feingold and M. Scheunert for correspondence and for pointing out useful references to us. JdB was sponsored in part by NSF grant 9309888, and LF by the AvH Stiftung. 


\section{Appendix A: Details on two quantum orbifolds}

This appendix contains the normal ordered versions of some classical relations for the orbifolds discussed in sect. 4 . The formulae below have been obtained on computer by using the OPE package of [31].

First we study the bosonic projection of the $N=1$ super Virasoro algebra. Conventions

are fixed by the following OPEs of the energy momentum tensor $\hat{L}$ and the spin $\frac{3}{2}$ fermion $\hat{G}$ :

$$
\begin{aligned}
& \hat{L}(z) \hat{L}(w)=\hbar\left(\frac{c / 2}{(z-w)^{4}}+\frac{2 \hat{L}(w)}{(z-w)^{2}}+\frac{\partial \hat{L}(w)}{z-w}\right)+\text { reg. } \\
& \hat{L}(z) \hat{G}(w)=\hbar\left(\frac{3 / 2 \hat{G}(w)}{(z-w)^{2}}+\frac{\partial \hat{G}(w)}{z-w}\right)+\text { reg. } \\
& \hat{G}(z) \hat{G}(w)=\hbar\left(\frac{2 c / 3}{(z-w)^{3}}+\frac{2 \hat{L}(w)}{z-w}\right)+\text { reg. . }
\end{aligned}
$$

In order to be able to compare with the classical case we have explicitly introduced the Planck constant $\hbar$ in (A.1). The normal ordered version of the simplest classical relation $\Phi^{1} \Phi^{1}=0$ becomes:

$$
\begin{aligned}
((\hat{G} \partial \hat{G})(\hat{G} \partial \hat{G}))=\hbar( & \frac{192 \hbar-31 c}{90}\left(\hat{G} \partial^{5} \hat{G}\right)+\frac{7(c+12 \hbar)}{18} \partial^{2}\left(\hat{G} \partial^{3} \hat{G}\right)-\frac{7(2 c+21 \hbar)}{72} \partial^{4}(\hat{G} \partial \hat{G}) \\
& -\frac{14}{3}\left(\left(\hat{G} \partial^{3} \hat{G}\right) \hat{L}\right)+\left((\hat{G} \partial \hat{G}) \partial^{2} \hat{L}\right)-3(\partial(\hat{G} \partial \hat{G}) \partial \hat{L})+3\left(\partial^{2}(\hat{G} \partial \hat{G}) \hat{L}\right) \\
& \left.-\hbar \frac{1}{6}\left(\hat{L} \partial^{4} \hat{L}\right)-\hbar \frac{7}{3}\left(\partial \hat{L} \partial^{3} \hat{L}\right)+\hbar \frac{41 c-771 \hbar}{540} \partial^{6} \hat{L}\right) .
\end{aligned}
$$

Apart from the quantized counterpart $\left(\hat{G} \partial^{5} \hat{G}\right)$ of the classical generator $\Phi^{5}$ on the r.h.s., eq. (A.2) is a polynomial in the energy momentum tensor $\hat{L}$ and the dimension 4 and dimension 6 generators $(\hat{G} \partial \hat{G})$ and $\left(\hat{G} \partial^{3} \hat{G}\right)$. Therefore, $\left(\hat{G} \partial^{5} \hat{G}\right)$ can be eliminated and does not give rise to a new generator. Note that the r.h.s. of (A.2) vanishes in the classical limit $\hbar \rightarrow 0$, as it should. Similarly, normal ordering the classical relation $\Phi^{3} \Phi^{1}=0$ leads to:

$$
\begin{aligned}
\left(\left(\hat{G} \partial^{3} \hat{G}\right)(\hat{G} \partial \hat{G})\right)=\hbar( & \frac{128 \hbar-25 c}{84}\left(\hat{G} \partial^{7} \hat{G}\right)+\frac{c+11 \hbar}{2} \partial^{2}\left(\hat{G} \partial^{5} \hat{G}\right)-\frac{20 c+161 \hbar}{24} \partial^{4}\left(\hat{G} \partial^{3} \hat{G}\right) \\
& +\frac{60 c+469 \hbar}{120} \partial^{6}(\hat{G} \partial \hat{G})-\frac{39}{10}\left(\left(\hat{G} \partial^{5} \hat{G}\right) \hat{L}\right)-6\left(\partial\left(\hat{G} \partial^{3} \hat{G}\right) \partial \hat{L}\right) \\
& +5\left(\partial^{2}\left(\hat{G} \partial^{3} \hat{G}\right) \hat{L}\right)+\frac{1}{2}\left((\hat{G} \partial \hat{G}) \partial^{4} \hat{L}\right)+\frac{7}{2}\left(\partial(\hat{G} \partial \hat{G}) \partial^{3} \hat{L}\right) \\
& \left.-\frac{5}{2}\left(\partial^{4}(\hat{G} \partial \hat{G}) \hat{L}\right)+\hbar \frac{4}{5}\left(\hat{L} \partial^{6} \hat{L}\right)+\hbar \frac{1}{5}\left(\partial \hat{L} \partial^{5} \hat{L}\right)-\hbar \frac{250 c+6897 \hbar}{3360} \partial^{8} \hat{L}\right) .
\end{aligned}
$$


Using (A.2) one can eliminate $\left(\hat{G} \partial^{5} \hat{G}\right)$ in (A.3). Apart from the term $\left(\hat{G} \partial^{7} \hat{G}\right)$ both sides of (A.3) are polynomials in the generators of scale dimension 2, 4 and 6 . This permits one to eliminate also $\left(\hat{G} \partial^{7} \hat{G}\right)$, which corresponds to the classical scale dimension 10 generator $\Phi^{7}$. Naturally, the r.h.s. of (A.3) vanishes in the limit $\hbar \rightarrow 0$. Finally, the normal ordered counterpart of (4.8) reads

$$
\begin{aligned}
\left((\hat{G} \partial \hat{G}) \partial^{2}(\hat{G} \partial \hat{G})\right)=\hbar( & \frac{8(16 \hbar-c)}{315}\left(\hat{G} \partial^{7} \hat{G}\right)+\frac{c+32 \hbar}{20} \partial^{2}\left(\hat{G} \partial^{5} \hat{G}\right)-\frac{5 c+6 \hbar}{12} \partial^{4}\left(\hat{G} \partial^{3} \hat{G}\right) \\
& +\frac{26 c+93 \hbar}{90} \partial^{6}(\hat{G} \partial \hat{G})-\frac{16}{15}\left(\left(\hat{G} \partial^{5} \hat{G}\right) \hat{L}\right)-\left(\left(\hat{G} \partial^{3} \hat{G}\right) \partial^{2} \hat{L}\right) \\
& -\frac{8}{3}\left(\partial\left(\hat{G} \partial^{3} \hat{G}\right) \partial \hat{L}\right)+\frac{5}{3}\left(\partial^{2}\left(\hat{G} \partial^{3} \hat{G}\right) \hat{L}\right)+\frac{1}{6}\left((\hat{G} \partial \hat{G}) \partial^{4} \hat{L}\right) \\
& -\frac{17}{6}\left(\partial(\hat{G} \partial \hat{G}) \partial^{3} \hat{L}\right)-3\left(\partial^{2}(\hat{G} \partial \hat{G}) \partial^{2} \hat{L}\right)-\frac{5}{6}\left(\partial^{3}(\hat{G} \partial \hat{G}) \partial \hat{L}\right) \\
& -\frac{3}{2}\left(\partial^{4}(\hat{G} \partial \hat{G}) \hat{L}\right)+\hbar \frac{28}{45}\left(\hat{L} \partial^{6} \hat{L}\right)+\hbar \frac{9}{5}\left(\partial \hat{L} \partial^{5} \hat{L}\right) \\
& \left.+\hbar \frac{7}{2}\left(\partial^{2} \hat{L} \partial^{4} \hat{L}\right)+\hbar \frac{89}{36}\left(\partial^{3} \hat{L} \partial^{3} \hat{L}\right)-\hbar \frac{322 c+13353 \hbar}{10080} \partial^{8} \hat{L}\right)
\end{aligned}
$$

After eliminating $\left(\hat{G} \partial^{5} \hat{G}\right)$ from (A.2) and $\left(\hat{G} \partial^{7} \hat{G}\right)$ from (A.3) the identity (A.4) is precisely the generic null field at scale dimension 10 in the bosonic projection of the quantum $N=1$ super Virasoro algebra.

The quantum version of the $\mathcal{W}(2,2)$ obtained from two commuting copies of the Virasoro algebra has the following OPEs:

$$
\begin{aligned}
\hat{L}(z) \hat{L}(w) & =\hbar\left(\frac{c / 2}{(z-w)^{4}}+\frac{2 \hat{L}(w)}{(z-w)^{2}}+\frac{\partial \hat{L}(w)}{z-w}\right)+\text { reg. } \\
\hat{L}(z) \hat{W}(w) & =\hbar\left(\frac{2 \hat{W}(w)}{(z-w)^{2}}+\frac{\partial \hat{W}(w)}{z-w}\right)+\text { reg. } \\
\hat{W}(z) \hat{W}(w) & =\hbar\left(\frac{c / 2}{(z-w)^{4}}+\frac{2 \hat{L}(w)}{(z-w)^{2}}+\frac{\partial \hat{L}(w)}{z-w}\right)+\text { reg. }
\end{aligned}
$$

where we have again kept the Planck constant. Now one can compute that the normal 
ordered counterpart of eq. (4.10) is:

$$
\begin{aligned}
& \partial^{2}((\hat{W} \hat{W})(\hat{W} \hat{W}))-6\left((\hat{W} \hat{W}) \partial^{2}(\hat{W} \hat{W})\right)+8\left((\hat{W} \hat{W})\left(\hat{W} \partial^{2} \hat{W}\right)\right)= \\
& \hbar\left(\frac{47 c-512 \hbar}{90}\left(\hat{W} \partial^{6} \hat{W}\right)-\frac{5(c+32 \hbar)}{4} \partial^{2}\left(\hat{W} \partial^{4} \hat{W}\right)+\frac{7 c-74 \hbar}{6} \partial^{4}\left(\hat{W} \partial^{2} \hat{W}\right)+\frac{37 \hbar-7 c}{30} \partial^{6}(\hat{W} \hat{W})\right. \\
& \quad+20\left(\left(\hat{W} \partial^{4} \hat{W}\right) \hat{L}\right)+36\left(\left(\hat{W} \partial^{2} \hat{W}\right) \partial^{2} \hat{L}\right)+28\left(\partial\left(\hat{W} \partial^{2} \hat{W}\right) \partial \hat{L}\right)-28\left(\partial^{2}\left(\hat{W} \partial^{2} \hat{W}\right) \hat{L}\right) \\
& \quad-4\left(\partial(\hat{W} \hat{W}) \partial^{3} \hat{L}\right)-20\left(\partial^{2}(\hat{W} \hat{W}) \partial^{2} \hat{L}\right)-\frac{44}{3}\left(\partial^{3}(\hat{W} \hat{W}) \partial \hat{L}\right)+4\left(\partial^{4}(\hat{W} \hat{W}) \hat{L}\right) \\
& \left.\quad+\hbar \frac{6}{5}\left(\hat{L} \partial^{6} \hat{L}\right)-\hbar \frac{9}{5}\left(\partial \hat{L} \partial^{5} \hat{L}\right)-\hbar \frac{8}{3}\left(\partial^{2} \hat{L} \partial^{4} \hat{L}\right)+\hbar \frac{1}{6}\left(\partial^{3} \hat{L} \partial^{3} \hat{L}\right)-\hbar \frac{184 c+38895 \hbar}{5040} \partial^{8} \hat{L}\right) .
\end{aligned}
$$

Using (A.6) one can express $\left(\hat{W} \partial^{6} \hat{W}\right)$ as a polynomial in the generators $\hat{L},(\hat{W} \hat{W}),\left(\hat{W} \partial^{2} \hat{W}\right)$ and $\left(\hat{W} \partial^{4} \hat{W}\right)$. This ensures that the $\mathbb{Z}_{2}$ orbifold of $\mathcal{W}(2,2)$ has no dimension 10 generator. Again, the r.h.s. of (A.6) vanishes in the limit $\hbar \rightarrow 0$ and it is not possible to eliminate this generator at the classical level.

In the case of $\mathcal{W}(2,2)$ we also would like to present a character argument indicating that one does not need new generators at higher dimensions either. Similarly to eq. (2.2), let $\phi_{2,4,6,8}(q)$ be the vacuum character of a $\mathcal{W}(2,4,6,8)$ without relations. It is straightforward to calculate the character $\chi_{0}(q)$ of the submodule of the $\mathcal{W}(2,2)$ vacuum module invariant under $\rho$. Up to order 29, we obtain for the difference of these two characters

$$
\begin{aligned}
\chi_{0}(q)-\phi_{2,4,6,8}(q)=-q^{12}(1 & +2 q+5 q^{2}+9 q^{3}+29 q^{5}+53 q^{6}+83 q^{7}+139 q^{8}+214 q^{9} \\
& +340 q^{10}+510 q^{11}+784 q^{12}+1153 q^{13}+1720 q^{14}+2491 q^{15} \\
& \left.+3634 q^{16}+5183 q^{17}+\mathcal{O}\left(q^{18}\right)\right) .
\end{aligned}
$$

All coefficients are nonpositive as expected. In particular, we read off from (A.7) that the first generic null field appears at scale dimension 12.

For more details on quantum orbifolds we refer the interested reader to [22]. 


\section{Appendix B: The invariants in the Hamiltonian reduction example}

In this appendix we show that the generating set of the ring $\mathcal{R}$ of differential polynomials in $L, I_{0}, Z_{+}, Z_{-}, I_{-}$invariant under the gauge group (5.7) is given by (5.11-14) as stated in section 5 . We shall do this by a construction which reduces the problem of finding the generating set of $\mathcal{R}$ to a problem in the invariant theory of the group $S L(2)$.

Note first that since $L$ does not mix with the other variables under the transformation (5.7), it can be factored out from the problem, i.e., $\mathcal{R}$ is generated by $L$ and the invariants depending on $I_{0}, Z_{+}, Z_{-}, I_{-}$. Similarly, $I_{0}$ can also be factored out if we introduce the new variables

$$
\tilde{L}:=L, \quad \tilde{I}_{0}:=I_{0}, \quad \tilde{Z}_{+}:=Z_{+}, \quad \tilde{Z}_{-}:=G Z_{-}, \quad \tilde{I}_{-}:=G I_{-},
$$

where $G$ is given by

$$
G(x):=\exp \left(-\frac{2}{\kappa} \int^{x} d t I_{0}(t)\right) .
$$

Indeed, in terms of these variables the gauge transformation rule (5.7) becomes

$$
\tilde{L} \rightarrow \tilde{L}, \quad \tilde{I}_{0} \rightarrow \tilde{I}_{0}, \quad \tilde{Z}_{+} \rightarrow \tilde{Z}_{+}, \quad \tilde{Z}_{-} \rightarrow \tilde{Z}_{-}-\tilde{\epsilon} \tilde{Z}_{+}, \quad \tilde{I}_{-} \rightarrow \tilde{I}_{-}+\kappa \partial \tilde{\epsilon},
$$

with

$$
\tilde{\epsilon}:=G \epsilon
$$

being arbitrary since $\epsilon$ in (5.7) was arbitrary.

Let $P\left(L, I_{0}, Z_{+}, Z_{-}, I_{-}\right)$be an arbitrary differential polynomial in the original variables and $\tilde{P}\left(\tilde{L}, \tilde{I}_{0}, \tilde{Z}_{+}, \tilde{Z}_{-}, \tilde{I}_{-}\right)$an arbitrary differential polynomial in the new variables. Decompose $P$ as a sum $\sum_{k} P_{k}$, where $P_{k}$ contains the terms of degree $k$, where we assign degree 1 to $Z_{-}$and $I_{-}$, and degree 0 to the other variables. Decompose $\tilde{P}$ as $\tilde{P}=\sum_{k} \tilde{P}_{k}$ in the same way. The map $F$ from tilded polynomials to untilded ones defined by

$$
F: \sum_{k} \tilde{P}_{k}\left(\tilde{L}, \tilde{I}_{0}, \tilde{Z}_{+}, \tilde{Z}_{-}, \tilde{I}_{-}\right) \mapsto \sum_{k} G^{-k} \tilde{P}_{k}\left(L, I_{0}, Z_{+}, G Z_{-}, G I_{-}\right)
$$

is invertibe, and the inverse $F^{-1}$ is given by

$$
F^{-1}: \sum_{k} P_{k}\left(L, I_{0}, Z_{+}, Z_{-}, I_{-}\right) \mapsto \sum_{k} G^{k} P_{k}\left(\tilde{L}, \tilde{I}_{0}, \tilde{Z}_{+}, G^{-1} \tilde{Z}_{-}, G^{-1} \tilde{I}_{-}\right) .
$$

This map naturally induces a one-to-one map between the respective invariant differential polynomials.

According to the above, it is enough to describe the differential polynomial invariants

in the tilded variables $\tilde{I}_{-}, \tilde{Z}_{+}, \tilde{Z}_{-}$under the transformation rule (B.3), which we now take in its (equivalent) infinitesimal form,

$$
\delta \tilde{I}_{-}=\kappa \partial \tilde{\epsilon}, \quad \delta \tilde{Z}_{+}=0, \quad \delta \tilde{Z}_{-}=-\tilde{\epsilon} \tilde{Z}_{+} .
$$


It is also convenient to introduce the notation

$$
I^{(l)}=\partial^{l} \tilde{I}_{-}, \quad \zeta_{-}^{(l)}:=\partial^{l} \tilde{Z}_{-}, \quad \zeta_{+}^{(l)}:=\partial^{l} \tilde{Z}_{+}, \quad \theta_{l}:=\partial^{l} \tilde{\epsilon},
$$

and let $I, \zeta_{+}, \zeta_{-}, \theta$ denote the corresponding infinite component vectors. Purely algebraically, the problem is to find the most general polynomial $\tilde{P}\left(\zeta_{-}, \zeta_{+}, I\right)$ which is is invariant under

$$
\delta I^{(l)}=\kappa \theta_{l+1}, \quad \delta \zeta_{+}^{(l)}=0, \quad \delta \zeta_{-}^{(l)}=-\sum_{m \geq 0}\left(\begin{array}{c}
l \\
m
\end{array}\right) \zeta_{+}^{(l-m)} \theta_{m}
$$

for arbitrary $\theta$. From computing the variation of $\tilde{P}$ using the chain rule, $\tilde{P}$ must satisfy

$$
\kappa \frac{\partial \tilde{P}}{\partial I^{(m-1)}}-\sum_{l \geq m}\left(\begin{array}{c}
l \\
m
\end{array}\right) \zeta_{+}^{(l-m)} \frac{\partial \tilde{P}}{\partial \zeta_{-}^{(l)}}=0, \quad\left(m \geq 0, \quad \frac{\partial \tilde{P}}{\partial I^{(-1)}}=0\right) .
$$

Consider now the decomposition of $\tilde{P}$ according to the different powers of $I$, given by ${ }^{6}$

$$
\tilde{P}=\sum_{r \geq 0} Q_{r} \quad \text { where } \quad Q_{r}\left(\zeta_{-}, \zeta_{+}, \lambda I\right)=\lambda^{r} Q_{r}\left(\zeta_{-}, \zeta_{+}, I\right)
$$

which leads to a refined form of (B.9), namely,

$$
\kappa \frac{\partial Q_{r+1}}{\partial I^{(m-1)}}-\sum_{l \geq m}\left(\begin{array}{c}
l \\
m
\end{array}\right) \zeta_{+}^{(l-m)} \frac{\partial Q_{r}}{\partial \zeta_{-}^{(l)}}=0, \quad(r=0,1, \ldots) .
$$

This implies that every invariant polynomial $\tilde{P}$ is uniquely determined by its $I$-independent term $Q_{0}$. On the other hand, taking $m=r=0$ in (B.11) we obtain that $Q_{0}$ is subject to

$$
\sum_{l \geq 0} \zeta_{+}^{(l)} \frac{\partial Q_{0}}{\partial \zeta_{-}^{(l)}}=0
$$

and the point is that this equation has a simple group theoretic meaning.

To see this take the standard action of the Lie algebra $s l(2)$, with generators $E, H, F$, on the variables $\zeta_{ \pm}^{(l)}$

$$
\begin{array}{ll}
\delta_{E} \zeta_{-}^{(l)}=\zeta_{+}^{(l)}, & \delta_{E} \zeta_{+}^{(l)}=0, \\
\delta_{F} \zeta_{+}^{(l)}=\zeta_{-}^{(l)}, & \delta_{F} \zeta_{-}^{(l)}=0, \\
\delta_{H} \zeta_{+}^{(l)}=\zeta_{+}^{(l)}, & \delta_{H} \xi_{-}^{(l)}=-\zeta_{-}^{(l)},
\end{array}
$$

and extend this by the Leibniz rule to an action of $\operatorname{sl}(2)$ on the ring of polynomials in the infinitely many doublets $\zeta_{ \pm}^{(l)}$. Clearly, (B.12) just defines the subring of 'highest weight polynomials', i.e., the kernel of $\delta_{E}$. Using the representation theory of $\operatorname{sl}(2)$ it is not hard

\footnotetext{
${ }^{6}$ The new letter $Q$ is used since this decomposition is different from that in (B.5).
} 
to see that the kernel of $\delta_{E}$ is generated by the pairwise symplectic scalar products of the different doublets and the highest weight components of the doublets themselves, given by

$$
w_{i, j}:=\zeta_{+}^{(i)} \zeta_{-}^{(j)}-\zeta_{-}^{(i)} \zeta_{+}^{(j)}, \quad \forall i \neq j, \quad \text { and } \quad \zeta_{+}^{(l)}, \quad \forall l
$$

(For instance, one can observe that the polynomials depending on a finite subset of the doublets and having definite degrees of homogeneity in those variables are an invariant subspace and using this one can show inductively that (B.14) indeed generates the kernel of $\left.\delta_{E}\right)$. One can also easily verify the relations

$$
\begin{gathered}
w_{i, j}+w_{j, i}=0, \\
w_{i, j} w_{k, l}-w_{i, k} w_{j, l}+w_{i, l} w_{j, k}=0, \\
w_{i, j} \zeta_{+}^{(k)}-w_{i, k} \zeta_{+}^{(j)}+w_{j, k} \zeta_{+}^{(i)}=0,
\end{gathered}
$$

which are analogous to (1.6-7) in section 1. Moreover, if we write

$$
\zeta_{+}^{(k)}=w_{k, \infty}=\zeta_{+}^{(k)} \zeta_{-}^{(\infty)}-\zeta_{-}^{(k)} \zeta_{+}^{(\infty)} \quad \text { with } \quad \zeta_{-}^{(\infty)}:=1, \zeta_{+}^{(\infty)}:=0
$$

and let the indices $r, s, p, q$ run over the nonnegative integers and $\infty$, then we can uniformly write (B.15-17) as

$$
\begin{gathered}
w_{r, s}+w_{s, r}=0 \\
w_{p, q} w_{r, s}-w_{p, r} w_{q, s}+w_{p, s} w_{q, r}=0 .
\end{gathered}
$$

Then we can apply the 'straightening algorithm' given in Chapter 3 of [13] to show that (B.19-20) imply all the relations satisfied by the generating set (B.14) of the ring of 'highest weight polynomials'. More precisely, this would be true if the variables $\zeta_{ \pm}^{(l)}$ were independent, but now we have the derivation $\partial \zeta_{ \pm}^{(l)}=\zeta_{ \pm}^{(l+1)}$, which implies the extra linear relation

$$
\partial w_{r, s}=w_{r+1, s}+w_{r, s+1} .
$$

At this point the solution space of (B.12) is fully under control and to derive the generating set of the gauge invariant differential polynomials all one has to do now is to follow the above construction backwards. First one solves the recursion relation (B.11) taking any of the elements in (B.14) for $Q_{0}$. Using also (B.7), this then yields the generating set of the invariants under (B.6). Then one returns to the original variables by means of (B.5). At the end of the day, one obtains $P_{i, j}$ given by (5.12) from $w_{i, j}$ in (B.14) by this procedure. Moreover, one can trace back the relations given by (5.13-14) to corresponding relations in (B.19-21). Since it is completely straightforward from here on, we omit the details of this derivation of the statement of section 5 . 


\section{References}

[1] P. Bouwknegt, K. Schoutens, W-Symmetry in Conformal Field Theory, Phys. Rep. 223 (1993) p. 183

[2] P. Bowcock, G.M.T. Watts, On the Classification of Quantum $\mathcal{W}$-Algebras, Nucl. Phys. B379 (1992) p. 63

[3] J. de Boer, T. Tjin, The Relation between Quantum $\mathcal{W}$ Algebras and Lie Algebras, preprint THU-93/05 (1993), IFTA-02-93, hep-th/9302006, to appear in Commun. Math. Phys.

[4] L. Fehér, L. O'Raifeartaigh, P. Ruelle, I. Tsutsui, On the Completeness of the Set of Classical $\mathcal{W}$-Algebras Obtained from DS Reductions, preprint BONN-HE-93-14 (1993), DIAS-STP-93-02, hep-th/9304125, to appear in Commun. Math. Phys.

[5] L. Fehér, L. O'Raifeartaigh, I. Tsutsui, The Vacuum Preserving Lie Algebra of a Classical $\mathcal{W}$-Algebra, Phys. Lett. B316 (1993) p. 275

[6] H.G. Kausch, G.M.T. Watts, A Study of $\mathcal{W}$-Algebras Using Jacobi Identities, Nucl. Phys. B354 (1991) p. 740

[7] W. Eholzer, A. Honecker, R. Hübel, How Complete is the Classification of $\mathcal{W}$ Symmetries?, Phys. Lett. B308 (1993) p. $42^{7}$

[8] K. Hornfeck, $\mathcal{W}$-Algebras with Set of Primary Fields of Dimensions $(3,4,5)$ and $(3,4,5,6)$, Nucl. Phys. B407 (1993) p. 237

[9] F. Delduc, L. Frappat, P. Sorba, F. Toppan, E. Ragoucy, Rational $\mathcal{W}$ Algebras From Composite Operators, Phys. Lett. B318 (1993) p. 457

[10] I. Bakas, E. Kiritsis, Beyond the Large $N$ Limit: Non-Linear $\mathcal{W}_{\infty}$ as Symmetry of the $S L(2, \mathbb{R}) / U(1)$ Coset Model, Int. Jour. Mod. Phys. A7, Suppl. 1A (1992) p. 55

[11] H. Weyl, The Classical Groups, Their Invariants and Representations, Princeton, New Jersey, Princeton University Press (1946)

[12] R. Howe, 'The Classical Groups' and Invariants of Binary Forms, Proc. Symposia Pure Mathematics 48 (1988), p. 133; Remarks on Classical Invariant Theory, Trans. AMS 313 (1989), p. 539

[13] J. Kung, G.-C. Rota, The Invariant Theory of Binary Forms, Bulletin of the AMS 10 (1984), p. 27

[14] F.A. Bais, P. Bouwknegt, M. Surridge, K. Schoutens, Extensions of the Virasoro Algebra Constructed from Kac-Moody Algebras Using Higher Order Casimir Invariants, Nucl. Phys. B304 (1988) p. 348

[15] P. Goddard, W. Nahm, D. Olive, Symmetric Spaces, Sugawara's Energy Momentum Tensor in Two Dimensions and Free Fermions, Phys. Lett. B160 (1985) p. 111

7 Note that a remark on the classification of 'exceptional' $\mathcal{W}$-algebras in this reference is slightly misleading because it was not recognized that $\mathcal{W}(2,8)$ at $c=-\frac{712}{7}$ and $c=-\frac{3164}{23}$ are related to minimal models of the Casimir algebras of $E_{8}$ and $E_{7}$ respectively. 
[16] J. de Boer, L. Fehér et al, in preparation

[17] P. Bouwknegt, Extended Conformal Algebras from Kac-Moody Algebras, Proceedings of the meeting 'Infinite dimensional Lie algebras and Groups', CIRM, Luminy, Marseille (1988) p. 527

[18] P. Goddard, A. Schwimmer, Unitary Construction of Extended Conformal Algebras, Phys. Lett. B206 (1988) p. 62

[19] P. Bowcock, P. Goddard, Coset Constructions and Extended Conformal Algebras, Nucl. Phys. B305 (1988) p. 685

[20] R. Blumenhagen, $\mathcal{W}$-Algebren in Konformer Quantenfeldtheorie, Diplomarbeit BONN-IR-91-06 (1991)

[21] J. Balog, L. Fehér, L. O'Raifeartaigh, P. Forgács, A. Wipf, Toda Theory and $\mathcal{W}$-Algebra from a Gauged WZNW Point of View, Ann. Phys. 203 (1990) p. 76

[22] R. Blumenhagen, W. Eholzer, A. Honecker, K. Hornfeck, R. Hübel, Cosets and Unifying $\mathcal{W}$-Algebras, BONN preprint, in preparation

[23] V.A. Fateev, A.B. Zamolodchikov, Nonlocal (Parafermion) Currents in Two-Dimensional Conformal Quantum Field Theory and Self-Dual Critical Points in $\mathbb{Z}_{N}$-Symmetric Statistical Systems, Sov. Phys. JETP 62 (1985) p. 215

[24] F.A. Bais, P. Bouwknegt, M. Surridge, K. Schoutens, Coset Construction for Extended Virasoro Algebras, Nucl. Phys. B304 (1988) p. 371

[25] V.A. Fateev, S.L. Lukyanov, The Models of Two-Dimensional Conformal Quantum Field Theory with $\mathbb{Z}_{n}$ Symmetry, Int. Jour. of Mod. Phys. A3 (1988) p. 507

[26] F. Ravanini, Informal Introduction to Extended Algebras and Conformal Field Theories with $c \geq 1$, preprint NORDITA-89/21P (1989)

[27] H.G. Kausch, Chiral Algebras in Conformal Field Theory, Ph.D. thesis, Cambridge University, September 1991

[28] A. Honecker, A Note on the Algebraic Evaluation of Correlators in Local Chiral Conformal Field Theory, preprint BONN-HE-92-25 (1992), hep-th/9209029

[29] L. Frappat, E. Ragoucy, P. Sorba, Folding the $\mathcal{W}$ Algebras, Nucl. Phys. B404 (1993) p. 805

[30] F. Gliozzi, J. Scherk, D. Olive, Supersymmetry, Supergravity Theories and the Dual Spinor Model, Nucl. Phys. B122 (1977) p. 253

[31] K. Thielemans, A Mathematica Package for Computing Operator Product Expansions, Int. Jour. Mod. Phys. C2 (1991) p. 787 